\title{
Biosynthesis of medicinal tropane alkaloids in yeast
}

https://doi.org/10.1038/s41586-020-2650-9

Received: 20 April 2020

Accepted: 23 July 2020

Published online: 2 September 2020

Check for updates
Prashanth Srinivasan ${ }^{1} \&$ Christina D. Smolke ${ }^{1,2 \bowtie}$

Tropane alkaloids from nightshade plants are neurotransmitter inhibitors that are used for treating neuromuscular disorders and are classified as essential medicines by the World Health Organization ${ }^{1,2}$. Challenges in global supplies have resulted in frequent shortages of these drugs ${ }^{3,4}$. Further vulnerabilities in supply chains have been revealed by events such as the Australian wildfires ${ }^{5}$ and the COVID-19 pandemic ${ }^{6}$. Rapidly deployable production strategies that are robust to environmental and socioeconomic upheaval ${ }^{7,8}$ are needed. Here we engineered baker's yeast to produce the medicinal alkaloids hyoscyamine and scopolamine, starting from simple sugars and amino acids. We combined functional genomics to identify a missing pathway enzyme, protein engineering to enable the functional expression of an acyltransferase via trafficking to the vacuole, heterologous transporters to facilitate intracellular routing, and strain optimization to improve titres. Our integrated system positions more than twenty proteins adapted from yeast, bacteria, plants and animals across six sub-cellular locations to recapitulate the spatial organization of tropane alkaloid biosynthesis in plants. Microbial biosynthesis platforms can facilitate the discovery of tropane alkaloid derivatives as new therapeutic agents for neurological disease and, once scaled, enable robust and agile supply of these essential medicines.
Tropane alkaloids (TAs) such as cocaine and atropine are present in plants from the nightshade (Solanaceae), coca (Erythroxylaceae) and bindweed (Convolvulaceae) families. Some TAs, including hyoscyamine and scopolamine, are used to treat neuromuscular disorders ranging from nerve agent poisoning to Parkinson's disease ${ }^{1,2}$. Direct chemical syntheses of TAs are not economically viable owing to challenging stereochemistries ${ }^{9}$. Thus, intensive cultivation of Duboisia shrubs from the nightshade family in Australia, India, Brazil and Saudi Arabia undergirds the global supply for medicinal TAs $s^{2,10,11}$. This agriculture-based supply chain poses three risks to public health. First, overall increasing demand for TA-based medicines already results in recurring supply shortages ${ }^{3,4}$. Second, regional events, such as the 2019-2020 Australian wildfires, can threaten global supply ${ }^{5}$. Third, global crises, such as the ongoing COVID19 pandemic, can threaten local availability owing to demand spikes and disruption to supply chains $s^{6,12}$. The urgency of having options for quickly scaling production of essential medicines to match regional and local demand, free of geopolitical dependencies and robust to environmental and socioeconomic upheaval, is widely recognized ${ }^{7,8}$.

Phytochemical production using engineered yeast can address many of the vulnerabilities associated with crop cultivation. The rapid generation times and high cell densities achieved in microbial fermentations enable production of target compounds with reduced time, space and resource requirements relative to plant extraction. Cultivation in closed bioreactors can also reduce supply chain susceptibility to environmental and geopolitical disruption, while providing improved batch-to-batch consistency and active ingredient purity.

However, biosynthesis of TAs in Solanaceae exhibits extensive intra- and intercellular compartmentalization, with enzymes active across specific sub-cellular compartments (cytosol, mitochondrion, chloroplast, peroxisome, ER membrane, vacuole), cell types (root pericycle, endodermis, cortex) and tissues (secondary roots) ${ }^{11}$. Reconstitution of such pathways in yeast is thus made challenging by incompatibilities of enzymes adapted for specific spatial or regulatory contexts, and metabolite transport strategies that are not readily realized in microbial hosts.

Hyoscyamine and scopolamine comprise an arginine-derived 8-azabicyclo[3.2.1]octane ('tropine') acyl acceptor esterified with a phenylalanine-derived phenyllactic acid (PLA) acyl donor (Fig. 1a). The identification of a type III polyketide synthase (PYKS) and cytochrome P450 (CYP82M3) catalysing the cyclization of $N$-methylpyrrolinium to tropinone in Atropa belladonna ${ }^{13}$ enabled us and others to engineer yeast strains for de novo production of tropine ${ }^{14,15}$. The recent report of a UDP-glucosyltransferase (UGT84A27) and serine carboxypeptidase-like (SCPL) acyltransferase (littorine synthase) catalysing the condensation of tropine and phenyllactate to littorine ${ }^{16}$ resolved a debate about the nature of the acyl transfer reaction ${ }^{9}$. However, functional expression of plant SCPL acyltransferases (SCPL-ATs) in non-plant hosts has not been reported. Also, although the cytochrome P450 (CYP80F1) that catalyses rearrangement of littorine to hyoscyamine aldehyde ${ }^{17,18}$ and the 2-oxoglutarate-dependent hydroxylase/dioxygenase $(\mathrm{H} 6 \mathrm{H})$ that catalyses epoxidation of hyoscyamine to scopolamine are established ${ }^{19,20}$, no enzymatic activity for reduction of hyoscyamine aldehyde to hyoscyamine is known, necessitating discovery of such an enzyme (Fig.1a).

\section{TA acyl acceptor and donor biosynthesis}

We designed a biosynthetic pathway comprising five functional modules for hyoscyamine and scopolamine production from simple 


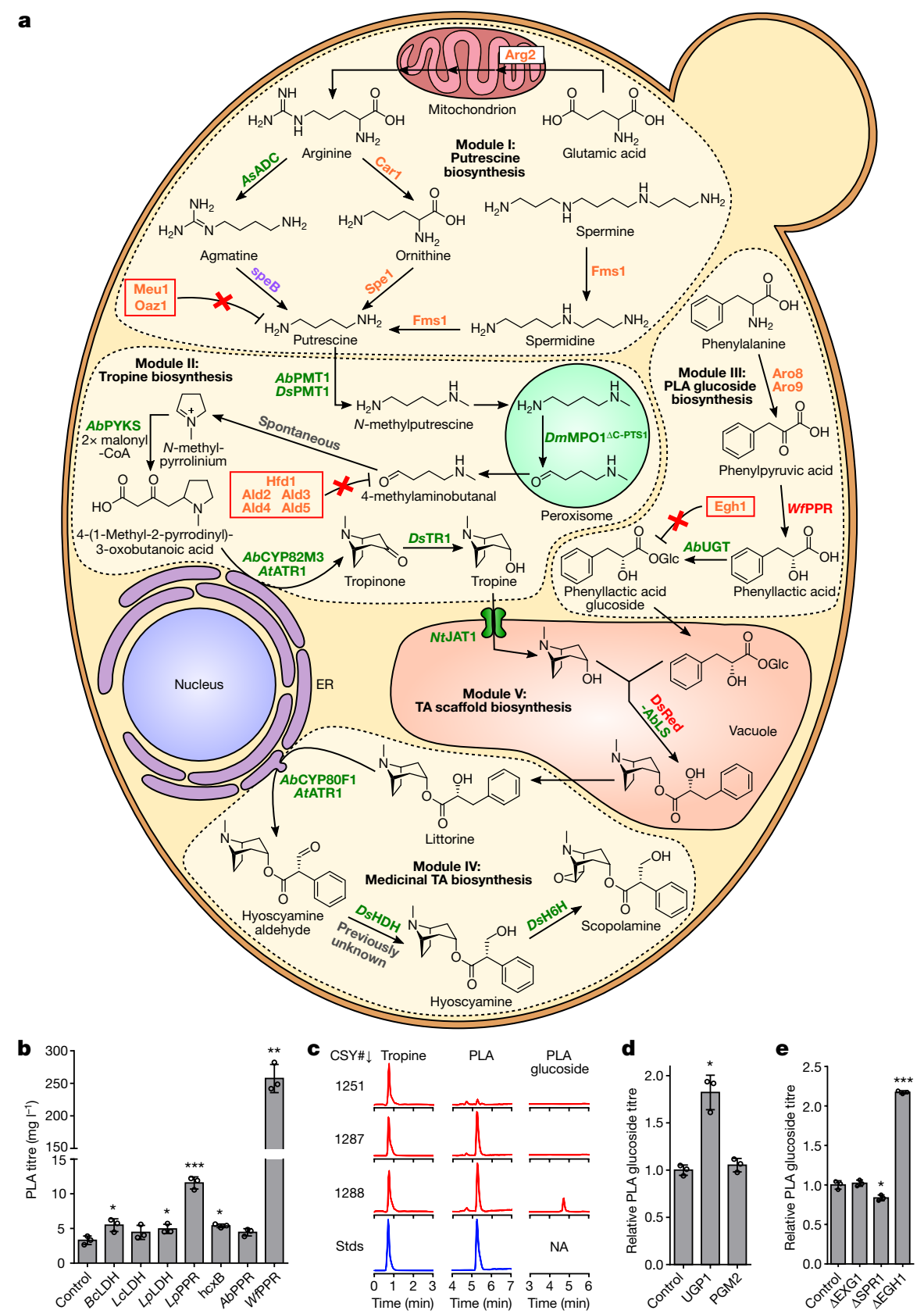

Fig. 1 Engineered biosynthetic pathway for de novo production of scopolamine in yeast and optimization of PLA-glucoside biosynthesis. a, Modular pathway construction for scopolamine biosynthesis in yeast. Enzyme/protein colour scheme: orange, yeast (overexpressed); green, plant; purple, bacteria; red, other eukaryote; grey, spontaneous/non-enzymatic. Red boxes indicate disrupted yeast proteins; dotted or solid lines of vacuole membrane delineate functional biosynthetic modules. DsRed-AbLS, Discosoma sp. red fluorescent protein fused to the $\mathrm{N}$ terminus of $A$. belladonna littorine synthase. $\mathbf{b}$, PLA production in yeast engineered for expression of PPRs or LDHs. Heterologous enzymes or negative control (BFP) were expressed from low-copy plasmids in strain CSY1251.c, Multiple reaction monitoring (MRM) and extracted ion chromatogram (EIC) traces from culture medium of yeast engineered for step-wise reconstitution of PLA glucoside

precursors in yeast (Fig. 1a). Modules I/II and III enable de novo biosynthesis of the acyl acceptor and donor moieties; module IV enables TA scaffold modifications to produce hyoscyamine and scopolamine; module V comprises the central acyltransferase reaction linking upstream acyl acceptor/donor biosynthesis to downstream scaffold modifications. As a starting point, we used a yeast platform strain (CSY1251) that was previously engineered for de novo production of the acyl acceptor biosynthesis via module III. Chromatogram traces are representative of three biological replicates. d, Relative titres of PLA glucoside in yeast engineered for overexpression of UDP-glucose biosynthetic enzymes. Enzymes or negative control (BFP) were expressed from low-copy plasmids in strain CSY1288.e, Relative PLA glucoside titres in CSY1288 with disruptions to endogenous glucosidases. In d and e, PLA glucoside accumulation was compared using relative titres owing to lack of an authentic chemical standard. Strains were cultured for $72 \mathrm{~h}$ before liquid chromatography-tandem mass spectrometry (LC-MS/MS) analysis of metabolites in culture supernatant. Data in $\mathbf{b}, \mathbf{d}$ and e represent the mean of $n=3$ biologically independent samples (open circles), error bars denote s.d. ${ }^{*} P<0.05,{ }^{* *} P<0.01$, ${ }^{* * *} P<0.001$, Student's two-tailed $t$-test. Statistical significance is shown relative to controls. Exact $P$ values are in Supplementary Table 5 .

tropine via modules I and II $^{14}$ (Extended Data Fig. 1). A putrescine biosynthesis module (I) designed to increase putrescine accumulation incorporated (i) overexpression of glutamate $\mathrm{N}$-acetyltransferase (Arg2), arginase (Car1), ornithine decarboxylase (Spe1) and polyamine oxidase (Fms1); (ii) a parallel plant/bacterial pathway encoded by Avena sativa arginine decarboxylase (AsADC) and Escherichia coli agmatine ureohydrolase (speB); and (iii) disruptions to polyamine regulatory 
mechanisms encoded by methylthioadenosine phosphorylase (Meu1) and ornithine decarboxylase antizyme-1 (Oaz1). A tropine biosynthesis module (II) incorporated (i) seven enzymes: A. belladonna and Datura stramonium putrescine $N$-methyltransferases (AbPMT1 and $D s$ PMT1), Datura metel $N$-methylputrescine oxidase engineered for improved peroxisomal activity $\left(D m \mathrm{MPO}^{\Delta \mathrm{C}-\mathrm{PTS1}}\right)$, A. belladonna pyrrolidine ketide synthase ( $A b$ PYKS) and tropinone synthase ( $A b C Y$ P82M3), Arabidopsis thaliana cytochrome $\mathrm{P} 450$ reductase (AtATR1) and D. stramonium tropinone reductase 1 (DsTR1); and (ii) disruptions to five aldehyde dehydrogenases (Hfd1, Ald2, Ald3, Ald4 and Ald5) to reduce loss of pathway intermediates.

We designed a third module (III) for production of the acyl donor 1- $O$ - $\beta$-phenyllactoylglucose (PLA glucoside) from phenylalanine via aromatic aminotransferases Aro8 and Aro9, phenylpyruvate reductase (PPR) and PLA UDP-glucosyltransferase (UGT84A27) ${ }^{16}$. Yeast produce 3-phenylpyruvate from phenylalanine via Aro8 and Aro $9^{21}$, and wild-type yeast and CSY1251 produce trace levels of PLA, potentially via nonspecific activity of a lactate dehydrogenase (LDH) acting on 3-phenylpyruvate ${ }^{22}$. We screened PPRs from E. coli ${ }^{23}$, Lactobacillus (UniProt AOA2U9AUW1), A. belladonna ${ }^{24}$ and Wickerhamia fluorescens ${ }^{25}$ and LDHs from Bacillus and Lactobacillus with reported activity on 3-phenylpyruvate ${ }^{22,26,27}$ via expression from a plasmid in CSY1251. All screened enzymes yielded modest (1.3- to 3.5-fold) improvements in PLA production relative to control, except for $W$. fluorescens PPR, which resulted in a nearly 80 -fold increase to approximately $250 \mathrm{mg} \mathrm{l}^{-1}$ (Fig. 1b) and was integrated into CSY1251 to make strain CSY1287.

In A. belladonna, PLA is activated for acyl transfer to tropine via glucosylation by UGT84A27 $(A b U G T)^{16}$. Plant UGTs participate in the biosynthesis of diverse phenylpropanoids and often exhibit broad substrate scope $^{28}$. We expressed $A b U G T$ from a plasmid in CSY1251 and measured conversion of three phenylpropanoid acyl donors (PLA, cinnamic acid and ferulic acid) to their respective glucosides (Extended Data Fig. 2a, c). Whereas $A b U$ UT glucosylated approximately $60 \%$ and $90 \%$ of cinnamic acid and ferulic acid, respectively, less than 3\% of PLA was glucosylated (Extended Data Fig. 2b). AbUGT orthologues identified from transcriptomes of other TA-producing Solanaceae (Supplementary Note1) and structure-guided active site mutants (Supplementary Note 2) exhibited poor activity on PLA (Extended Data Fig. 2b, d-f), which suggests that PLA glucosylation may constitute a key limitation in TA production. We constructed strain CSY1288 by integrating codon-optimized $W$. fluorescens 3-phenylpyruvate reductase (WfPPR) and $A b U G T$ into the genome of CSY1251, and verified PLA production $\left(66 \mathrm{mg} \mathrm{l}^{-1}\right)$ and minimal PLA glucoside accumulation (Fig. 1c).

We increased PLA glucoside levels by incorporating genetic modifications that promote UDP-glucose accumulation and decrease glycoside degradation. We overexpressed the PGM2 and UGP1 genes, which encode proteins that catalyse the isomerization of glucose-6-phosphate to glucose-1-phosphate and the conversion of glucose-1-phosphate to UDP-glucose, respectively, from plasmids in CSY1288. Although overexpression of $P G M 2$ resulted in no improvement relative to control, overexpression of $U G P 1$ resulted in an approximately 1.8-fold increase in the production of PLA glucoside (Fig.1d). We disrupted three native glucosidase genes-EXG1,SPR1 and EGH1-in CSY1288, as glucosidases have been shown to hydrolyse heterologous glucosides in yeast ${ }^{29}$. The disruption of $E G H 1$ more than doubled PLA glucoside production (Fig. 1e), indicating that hydrolysis by Egh1 (steryl- $\beta$-glucosidase) constitutes a substantial loss of TA precursor from the pathway. We thus incorporated both UGP1 overexpression and $E G H 1$ disruption into a complete TA production strain.

\section{HDH discovery and scopolamine biosynthesis}

We used a functional genomics approach to discover the enzyme, hyoscyamine dehydrogenase $(\mathrm{HDH})$, which catalyses the reduction
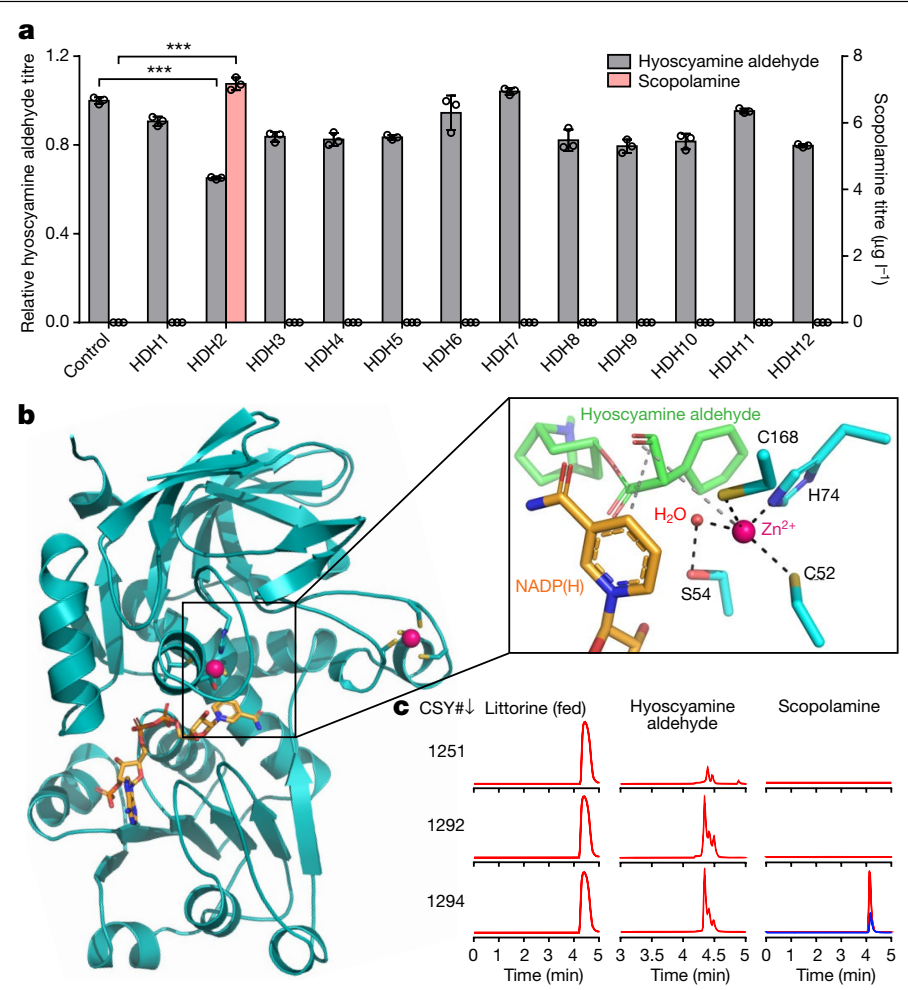

Fig. 2 | Identification and characterization of hyoscyamine dehydrogenase in A. belladonna. a, Production of hyoscyamine aldehyde and scopolamine in yeast engineered for expression of $A$. belladonna $\mathrm{HDH}$ candidates. Candidates or a negative control (BFP) were expressed from low-copy plasmids in CSY1292. Accumulation of hyoscyamine aldehyde was compared using relative titres owing to lack of an authentic chemical standard. Amino acid sequences are in Supplementary Table 1 . Data represent the mean of $n=3$ biologically independent samples (open circles), error bars denote s.d. ${ }^{*} P<0.05,{ }^{* *} P<0.01$, ${ }^{* * *} P<0.001$, Student's two-tailed $t$-test. Statistical significance is shown relative to control. Exact $P$ values are in Supplementary Table 5. b. Homology model of $A b \mathrm{HDH}$. NADPH and $\mathrm{Zn}^{2+}$ are shown in orange and pink, respectively. Box shows magnified view of $A b \mathrm{HDH}$ active site with NADPH and docked hyoscyamine aldehyde. Dashed lines indicate interactions important for catalysis. c, MRM traces from culture media of yeast engineered for step-wise reconstitution of module IV for conversion of littorine to scopolamine. Blue trace represents $125 \mathrm{nM}\left(38 \mathrm{\mu g} \mathrm{l}^{-1}\right)$ scopolamine standard. Chromatogram traces are representative of three biological replicates. In a and c, strains were cultured for $72 \mathrm{~h}$ with $1 \mathrm{mM}$ littorine before LC-MS/MS analysis of metabolites in culture supernatant.

of hyoscyamine aldehyde to hyoscyamine. We searched for genes that co-express with TA biosynthetic genes in secondary root tissues by mining a publicly available $A$. belladonna transcriptome dataset ${ }^{30}$. Starting from more than 40,000 identified transcripts, we removed transcripts without putative dehydrogenase or reductase-like domains, and further filtered by clustering tissue-specific expression profiles with those of bait genes $A b C Y P 80 F 1$ (littorine mutase) and $A b \mathrm{H} 6 \mathrm{H}$ (Extended Data Fig. 3a). Nearly all candidates exhibited the secondary root-specific expression pattern observed for TA biosynthetic genes. Owing to missing sequence regions, we repeated the de novo transcriptome assembly from raw RNA sequencing (RNA-seq) reads ${ }^{30}$ using the Trinity software package ${ }^{31}$ and reconstituted missing fragments for 12 $\mathrm{HDH}$ candidates via alignment of incomplete regions against the newly assembled transcriptome (Supplementary Table 1).

We identified the missing HDH activity by screening candidates generated via transcriptome mining in yeast. Lack of an authentic commercial standard for hyoscyamine aldehyde and insufficient yield from chemical syntheses, as well as similar chromatographic and mass spectrometric properties of littorine and hyoscyamine, necessitated screening of HDH candidates by detection of scopolamine $\left(\mathrm{m} / z 304[\mathrm{M}+\mathrm{H}]^{+}\right)$ 
from fed littorine $\left(\mathrm{m} / z 290[\mathrm{M}+\mathrm{H}]^{+}\right)$via a three-step biosynthetic pathway (Fig. 1a). We constructed an HDH screening strain (CSY1292) by integrating codon-optimized $A b C Y P 80 F 1$ and an optimal $\mathrm{H} 6 \mathrm{H}$ orthologue from D. stramonium (DsH6H) (Extended Data Fig. 4) into the genome of CSY1251, and expressed codon-optimized HDH candidates from a plasmid. One of the candidates, $\mathrm{HDH} 2$ (that is, $A b \mathrm{HDH}$ ), exhibited a 35\% decrease in hyoscyamine aldehyde levels and accumulation of scopolamine (7.2 $\left.\mathrm{g} \mathrm{I}^{-1}\right)$, indicating the missing HDH activity (Fig. 2a).

Structural and phylogenetic analyses provided insight into the catalytic mechanism and evolutionary history of HDH (Supplementary Notes 3,4$)$. Homology modelling indicated that $A b \mathrm{HDH}$ is a zinc-dependent alcohol dehydrogenase of the medium-chain dehydrogenase/reductase (MDR) superfamily and probably uses NADPH as the hydride donor for hyoscyamine aldehyde reduction (Fig. 2b, Supplementary Note 3). Ligand docking simulations and active site mutants suggested a mechanism in which the oxyanion intermediate formed upon hydride attack of hyoscyamine aldehyde is stabilized by a catalytic $\mathrm{Zn}^{2+}$, which is bound by Cys52, His74, Cys168 and a displaceable water molecule positioned by polar interactions with Ser54 (Fig. 2b, Extended Data Fig. 3b, Supplementary Note 3). We identified orthologues of $A b \mathrm{HDH}$ from transcriptomes of Datura innoxia (DiHDH) and D. stramonium $(D s \mathrm{HDH})^{32}$, and verified their activity via co-expression with an additional copy of $D s \mathrm{H} 6 \mathrm{H}$ from plasmids in CSY1292. DsHDH showed the highest substrate depletion and product accumulation of the variants tested (Extended Data Fig. 3c, d).

We reconstituted the medicinal TA biosynthetic branch (module IV) comprising optimal enzyme variants and overexpression of a limiting enzyme into our platform strain. Strain CSY1294 was constructed by integrating codon-optimized WfPPR and $A b U G T$ (module III), DsHDH, and an additional copy of $D s \mathrm{H} 6 \mathrm{H}$, which limits scopolamine accumulation (Extended Data Fig.3d), into CSY1292.Scopolamine production from fed littorine was verified in CSY1294 (Fig. 2c). Strain CSY1294 incorporates the enzymes for producing the acyl acceptor (tropine; modules I/II) and acyl donor (PLA glucoside; module III) for littorine biosynthesis, and the enzymes for modification of the TA scaffold to scopolamine (moduleIV), leaving the central acyltransferase reaction catalysed by littorine synthase (module V) as the final enzymatic step to implement.

\section{Engineering vacuolar littorine biosynthesis}

Recently, littorine biosynthesis in A. belladonna was demonstrated to occur via esterification of glucosylated PLA with tropine by an acyltransferase of the SCPL family (littorine synthase, $A b \mathrm{LS}$ ) ${ }^{16}$. Few plant SCPL-ATs have been studied and no reports of in vivo activity in non-plant hosts have emerged, owing to difficulties of extensive post-translational processing and trafficking in microbial hosts ${ }^{33}$.SCPL-ATs are expressed via the secretory pathway and localize to the plant tonoplast ${ }^{33}$ (Extended Data Fig. 6a). An N-terminal signal peptide directs the nascent polypeptide to the ER, where it undergoes processing steps for foldingsignal peptide cleavage, disulfide bond formation, and, in some cases, proteolytic removal of propeptide sequences. The partially folded SCPL-AT protein is transported through the Golgi, where it acquires $\mathrm{N}$-glycosylation on asparagine residues within $\mathrm{N}-\mathrm{X}$-S/T motifs (in which $\mathrm{X}$ is not proline). Recognition of cryptic signal sequences by vacuole-associated transport factors directs SCPL-AT to the vacuole lumen ${ }^{34}$. Although the yeast secretory pathway possesses much the same compartments and processing steps as in plants, it is unlikely that yeast transport factors recognize the same signal sequences and yeast protein glycosylation patterns differ from those of plants ${ }^{35}$. Our initial attempts to express wild-type $A b$ LS in CSY1294 resulted in a severe growth defect and no detectable TA biosynthesis.

We then showed that terminal and internal peptide sequences impact processing and localization of SCPL-ATs in yeast. A putative N-terminal signal peptide in $A b$ LS suggested that it follows the expected SCPL-AT ER-to-vacuole trafficking pathway in planta.
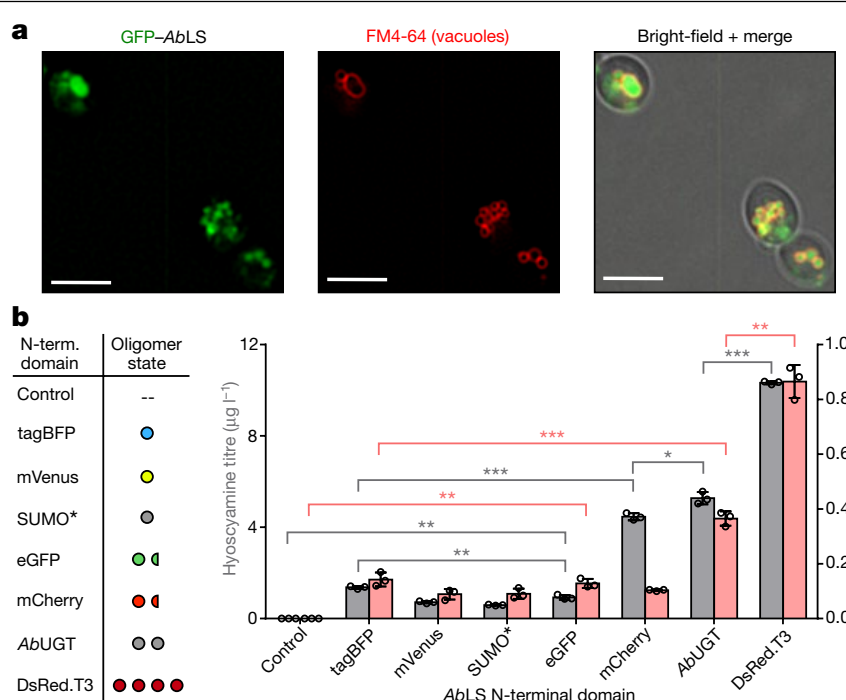

b

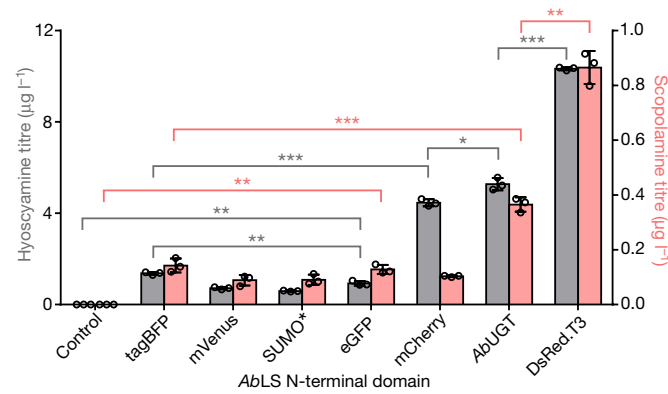

Fig. 3 | Engineering littorine synthase for activity in yeast. a, Yeast epifluorescence microscopy showing N-terminal GFP-tagged $A b$ LS (GFP- $A b \mathrm{LS}$ ), vacuolar membrane stain FM4-64, and bright-field merged images. Microscopy was performed on CSY1294 expressing GFP- $A b$ LS from a low-copy plasmid. 2D deconvolution was performed as described in the Methods. Scale bar, $5 \mu \mathrm{m}$. Images are representative of two independent experiments. b, De novo hyoscyamine and scopolamine production in engineered yeast expressing $A b$ LS N-terminal fusions. Table shows expected oligomerization state of each $\mathrm{N}$-terminal domain; half-circles (eGFP, mCherry) indicate monomer/weak dimer. Wild-type (control) or $A b \mathrm{LS}$ fusions were expressed from low-copy plasmids in CSY1294. Transformed strains were cultured for $96 \mathrm{~h}$ before LC-MS/MS analysis of metabolites in culture supernatant. No littorine was detected, indicating complete conversion to downstream TAs. Data represent the mean of $n=3$ biologically independent samples (open circles), error bars denote s.d. ${ }^{*} P<0.05,{ }^{* *} P<0.01,{ }^{* * *} P<0.001$, Student's two-tailed $t$-test. Exact $P$ values are in Supplementary Table 5.

Fluorescence microscopy of $\mathrm{N}$ - and C-terminal green fluorescent protein (GFP) fusions of $A b$ LS expressed from plasmids in CSY1294 revealed that the $\mathrm{N}$-terminal fusion (GFP- $A b \mathrm{LS}$ ) co-localized with a vacuolar membrane stain (Fig. 3a, Extended Data Fig. 6b), whereas no fluorescence was detected for the C-terminal fusion ( $A b$ LS -GFP), consistent with reports that a native $C$ terminus is crucial for SCPL-AT folding ${ }^{36}$. To identify possible failure points in $A b$ LS expression, maturation and trafficking in yeast, we screened $A b$ LS variants engineered for localization to subcellular compartments (Supplementary Note 5) and compared $A b \mathrm{LS} \mathrm{N}$-glycosylation patterns in tobacco and yeast (Extended Data Fig. 6c-h, Supplementary Note 6), which did not implicate mis-targeting or mis-glycosylation as primary factors impeding activity in yeast. Characterization of $A b$ LS endoproteolytic processing based on identification of a putative internal propeptide sequence suggested that the enzyme may become stalled in the yeast secretion pathway upstream of the trans-Golgi network (TGN) (Extended Data Figs. 6g, h, 7, Supplementary Note 7). This potential disruption of TGN sorting may account for the lack of activity and growth defect observed in CSY1294 expressing wild-type $A b$ LS.

Functional expression of $A b \mathrm{LS}$ in yeast was achieved by engineering $\mathrm{N}$-terminal fusions that may alter sorting from the TGN. Transport of soluble proteins from the TGN to the vacuole requires recognition of a typically $\mathrm{N}$-terminal signal sequence by vacuole protein sorting (Vps) cargo transport proteins, whereas integral membrane proteins that reach the yeast TGN are sorted to the vacuole by default ${ }^{37,38}$. We hypothesized that conversion of $A b$ LS into a transmembrane protein by masking the signal peptide with an $\mathrm{N}$-terminally fused soluble domain might resolve the putative obstruction in TGN sorting (Fig. 3a, Supplementary Note 8 ). We constructed $A b$ LS variants with N-terminally fused soluble domains, including fluorescent proteins from Aequoria 
(eGFP, tagBFP, mVenus) and Discosoma (mCherry, DsRed.T3); small ubiquitin-related modifier ( $\mathrm{Smt} 3$ ) with a mutated protease cleavage site $\left(\mathrm{SUMO}^{*}\right)^{39}$; and $A b \mathrm{UGT}$. We expressed these variants and wild-type $A b \mathrm{LS}$ from plasmids in CSY1294. Enhancement of $A b \mathrm{LS}$ activity appeared to be correlated with the $\mathrm{N}$-terminal domain oligomerization state, with scopolamine production increasing from monomeric or weakly dimeric (GFP, BFP, mVenus, mCherry and SUMO*) to homodimeric (AbUGT) and homotetrameric (DsRed) domains; reaching de novo hyoscyamine and scopolamine titres up to $10.3 \mu \mathrm{g} \mathrm{I}^{-1}$ and $0.87 \mu \mathrm{g} \mathrm{l}^{-1}$, respectively (Fig. 3b). To generate a strain containing all five metabolic modules for complete TA biosynthesis (modules I-V) (Fig. 1a), we integrated a codon-optimized DsRed- $A b$ LS and an additional copy of $U G P 1$ into the genome of CSY1294 at the disrupted $E G H 1$ site to generate CSY1296. CSY1296 exhibited de novo hyoscyamine and scopolamine titres of $10.2 \mu \mathrm{gl}^{-1}$ and $1.0 \mu \mathrm{gl}^{-1}$, respectively.

Inter-compartment transport limitations were addressed by incorporation of plant transporters. Vacuolar compartmentalization of DsRed- $A b$ LS in CSY1296 (Extended Data Fig. 8) necessitates import of cytosolic tropine and PLAglucoside to the vacuole lumen and export of vacuolar littorine to the cytosol. Several multidrug and toxin extrusion (MATE) transporters responsible for vacuolar alkaloid and glycoside sequestration have been identified in Solanaceae, including three with observed or predicted activity on $\mathrm{TAs}^{40,41}$. We expressed Nicotiana tabacum jasmonate-inducible alkaloid transporter 1 (NtJAT1) and two MATEs (NtMATE1, NtMATE2) from plasmids in CSY1296. Expression of NtJAT1 and NtMATE2 improved TA production; the former resulting in $74 \%$ and $18 \%$ increases in hyoscyamine and scopolamine titres, respectively (Fig. 4a). Fluorescence microscopy of CSY1296 expressing C-terminal GFP fusions of $N t$ JAT1 or NtMATE2 from plasmids supports the hypothesis that NtJAT1 localizes to the vacuolar membrane (co-localizing with DsRed-AbLS), whereas NtMATE2 is partitioned between vacuolar and plasma membranes (Extended Data Fig. 8), which suggests that both transporters might function to alleviate vacuolar substrate transport limitations while the latter might also improve cellular TA export (Fig. 4b).

Improvements in TA production were achieved via overexpression of limiting enzymes and media optimization. Additional copies of WfPPR and DsH6H expressed from plasmids in CSY1296 resulted in $64 \%$ and $89 \%$ increases in hyoscyamine and scopolamine titres, respectively (Extended Data Fig. 9). Supplementation with iron and 2-oxoglutarate (2-OG), required for $\mathrm{H} 6 \mathrm{H}$ activity ${ }^{19,42}$, resulted in 9.0- and 3.4-fold increases in hyoscyamine and scopolamine titres from CSY1296 (Fig. 4c). We constructed an optimized strain (CSY1297) by integrating NtJAT1 and additional copies of WfPPR and DsH6H into CSY1296, which showed 2.4- and 7.1-fold respective increases in hyoscyamine and scopolamine accumulation (Fig. 4c). Removing leucine auxotrophy by expressing 3-isopropylmalate dehydrogenase (Leu2) from a plasmid in CSY1297 (denoted CSY1298) increased conversion of hyoscyamine (85\% decrease) to scopolamine (more than 3 -fold increase) (Fig. 4c), potentially by improving access to $\mathrm{Fe}^{2+}$ via increased $\mathrm{NADH}$ regeneration ${ }^{43}$. Pseudo-fed-batch, high-density, shake-flask cultures grown in optimized media showed nolittorine accumulation, hyoscyamine and scopolamine titres of approximately $30 \mathrm{\mu g} \mathrm{I}^{-1}$ in CSY1297 and CSY1298, respectively, and tropine and PLA accumulation up to $3 \mathrm{mgl}^{-1}$ and $160 \mathrm{mgl}^{-1}$ (Extended Data Fig. 10, Supplementary Note 9), suggesting incorporation of PLA into littorine is a major limitation and target for future improvement.

\section{Discussion}

Our final strain comprises 34 chromosomal modifications (26 genes, 8 gene disruptions), resulting in an integrated whole-cell system that expresses enzymes and transporters in diverse sub-cellular locations (cytosol, mitochondria, peroxisome, vacuole, ER and vacuolar membranes)(Supplementary Note10). Combining functional genomics with our synthesis platform, we identified an oxidoreductase that catalyses
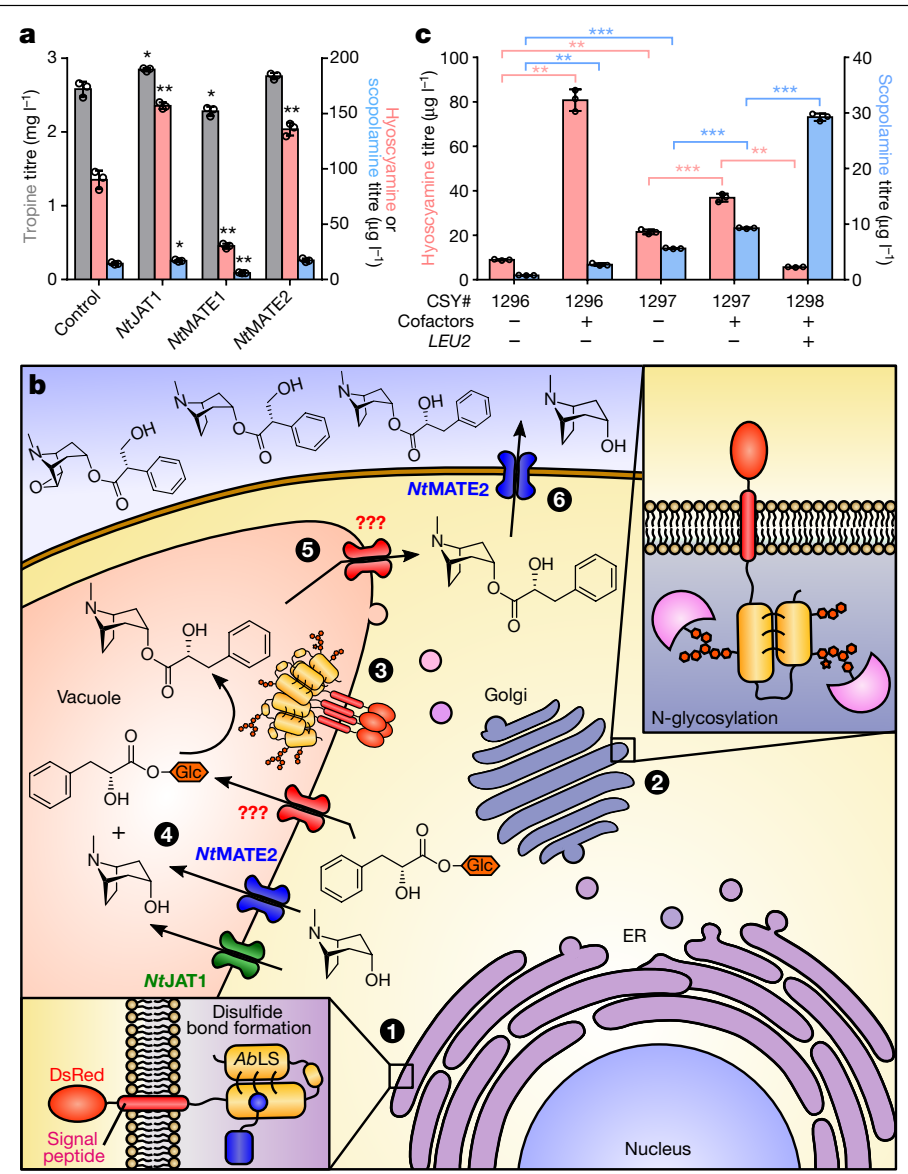

Fig. 4| Optimization of substrate transport limitations and medicinal TA production. a, Production of tropine, hyoscyamine and scopolamine in CSY1296 engineered for expression of heterologous alkaloid transporters. NtJAT1, MATE transporters $1 / 2$, or a negative control (BFP) were expressed from low-copy plasmids in CSY1296 and transformed strains were cultured for $96 \mathrm{~h} . \mathbf{b}$, Illustration of proposed DsRed- $A b$ LS trafficking and alleviation of substrate transport limitations via heterologous transporter expression in engineered yeast. Putative transport activities based on microscopy studies are indicated; '???' indicates unknown transport mechanism. Circled numbers indicate major proposed steps in DsRed- $A b \mathrm{LS}$ expression and activity, including maturation in (1) ER lumen and (2) Golgi, (3) trafficking to vacuole membrane, vacuolar (4) substrate import and (5) product export and (6) cellular TA export.c, Summary of strain and media optimization for de novo scopolamine production in engineered yeast. Strains were cultured in non-selective (CSY1296, CSY1297) or selective (CSY1298: leucine dropout) medium with or without cofactors $\left(50 \mathrm{mM} 2\right.$-oxoglutarate, $\left.15 \mathrm{mgl}^{-1} \mathrm{Fe}^{2+}\right)$ at $25^{\circ} \mathrm{C}$ for $96 \mathrm{~h}$. Strain CSY1298 is prototrophic for leucine and contains a blank plasmid with the LEU2gene (pCS4213). In a and c, metabolite titres in culture supernatant were quantified by LC-MS/MS. Data indicate the mean of $n=3$ biologically independent samples (open circles), error bars denotes.d. ${ }^{*} P<0.05,{ }^{* *} P<0.01,{ }^{* * *} P<0.001$, Student's two-tailed $t$-test. Exact $P$ values are in Supplementary Table 5 .

the remaining uncharacterized step in the biosynthesis of hyoscyamine and scopolamine. We developed an $\mathrm{N}$-terminal fusion strategy to achieve functional expression of the key TA scaffold-generating enzyme $A b$ LS. Our strategy may improve folding and trafficking of the engineered transmembrane $A b \mathrm{LS}$ through the secretory pathway to the vacuole (Supplementary Note 8), potentially enabling heterologous expression of plant SCPL-ATs and expanding the diversity of natural product biosyntheses in yeast ${ }^{33}$. We used a plant vacuolar alkaloid importer to address import restrictions in that compartment. Although plasma membrane transporters have been used to improve cellular export and import of metabolites ${ }^{44,45}$, our work demonstrates that incorporation of plant transporters can facilitate intracellular transport and help reconstruct sub-cellular compartmentalization inherent to many plant biosynthetic pathways. 
Our demonstration of total biosynthesis of hyoscyamine and scopolamine via engineered yeast suggests that centralized, plantation-based supply of medicinal TAs can be complemented or replaced by industrial fermentation. Process improvements to increase productivities from titres reported here (around 30 to $80 \mathrm{\mu g}^{-1}$ ) (Fig. 4c), which are typical of first implementations of complex plant natural product pathways ${ }^{46-48}$, to commercial production (approximately $5 \mathrm{gl}^{-1}$ ) are becoming routine ${ }^{49}$, and we anticipate would take 1-2 years of focused effort by a professional team (Supplementary Note 11). From a land-use perspective, we estimate that a fermentation-based process sourcing sugar from sugarcane would require at least 10 -fold less land than the existing Duboisia farming-based approach (Supplementary Note 12). Transitioning from agriculture-to fermentation-based production could have many indirect effects ranging from land-use and natural biodiversity, to labour markets and livelihoods, to supply-chain decouplings and geopolitical interdependencies ${ }^{50}$. Practically, because a fermentation-based approach can be implemented where needed and operated with a process time of days, our results support development of flexible manufacturing platforms enabling robust and agile supply of essential medicines.

\section{Online content}

Any methods, additional references, Nature Research reporting summaries, source data, extended data, supplementary information, acknowledgements, peer review information; details of author contributions and competing interests; and statements of data and code availability are available at https://doi.org/10.1038/s41586-020-2650-9.

1. World Health Organization. WHO Model List of Essential Medicines - 19th List (April 2015)

2. Grynkiewicz, G. \& Gadzikowska, M. Tropane alkaloids as medicinally useful natural products and their synthetic derivatives as new drugs. Pharmacol. Rep. 60, 439-463 (2008).

3. U.S. Food and Drug Administration. FDA Drug Shortages: Atropine Sulfate Injection (FDA Drug Shortages database, accessed online 14 April 2020); https://www.accessdata.fda. gov/scripts/drugshortages/dsp_ActivelngredientDetails.cfm?Al=AtropineSulfatelnjection \&st=c.

4. U.S. Food and Drug Administration. FDA Drug Shortages: Scopolamine Transdermal System (FDA Drug Shortages database, accessed online 14 April 2020); https://www. accessdata.fda.gov/scripts/drugshortages/dsp_ActivelngredientDetails.cfm?Al=Scopola mineTransdermalSystem\&st=r.

5. The Climate Council of Australia. 'This is Not Normal': Climate Change and Escalating Bushfire Risk https://www.climatecouncil.org.au/wp-content/uploads/2019/11/CC-novBushfire-briefing-paper.pdf (2019).

6. Hahn, S. M. FDA Statement: Coronavirus (COVID-19) Supply Chain Update https://www. fda.gov/news-events/press-announcements/coronavirus-covid-19-supply-chain-update (2020).

7. U.S. Food and Drug Administration. Coronavirus (Covid-19) Update: FDA Takes Further Steps to Help Mitigate Supply Interruptions of Food and Medical Products https://www. fda.gov/news-events/press-announcements/coronavirus-covid-19-update-fda-takesfurther-steps-help-mitigate-supply-interruptions-food-and (2020).

8. Agrawal, G., Ahlawat, H. \& Dewhurst, M. Winning Against COVID-19: the Implications for Biopharma https://www.mckinsey.com/industries/pharmaceuticals-and-medicalproducts/our-insights/winning-against-covid-19-the-implications-for-biopharma?from= groupmessage\&isappinstalled=0\# (2020)

9. Kohnen, K. L., Sezgin, S., Spiteller, M., Hagels, H. \& Kayser, O. Localization and organization of scopolamine biosynthesis in Duboisia myoporoides R. Br. Plant Cell Physiol. 59, 107-118 (2018).

10. Ullrich, S. F., Hagels, H. \& Kayser, O. Scopolamine: a journey from the field to clinics. Phytochem. Rev. 16, 333-353 (2017).

11. Kohnen-Johannsen, K. L. \& Kayser, O. Tropane alkaloids: chemistry, pharmacology, biosynthesis and production. Molecules 24, 1-23 (2019).

12. American Society of Anesthesiologists (ASA). ASA Urges Federal Government to Take Action on Drug Shortages https://www.asahq.org/advocacy-and-asapac/fda-andwashington-alerts/washington-alerts/2020/04/asa-urges-federal-government-to-takeaction-on-drug-shortages (2020).

13. Bedewitz, M. A., Jones, A. D., D’Auria, J. C. \& Barry, C. S. Tropinone synthesis via an atypical polyketide synthase and P450-mediated cyclization. Nat. Commun. 9, 5281 (2018).

14. Srinivasan, P. \& Smolke, C. D. Engineering a microbial biosynthesis platform for de novo production of tropane alkaloids. Nat. Commun. 10, 3634 (2019).

15. Ping, Y. et al. De novo production of the plant-derived tropine and pseudotropine in yeast. ACS Synth. Biol. 8, 1257-1262 (2019).

16. Qu, F. et al. Functional genomics analysis reveals two novel genes required for littorine biosynthesis. New Phytol. 225, 1906-1914 (2019).

17. Li, R. et al. Functional genomic analysis of alkaloid biosynthesis in Hyoscyamus niger reveals a cytochrome $\mathrm{P} 450$ involved in littorine rearrangement. Chem. Biol. 13 513-520 (2006)
18. Nasomjai, P. et al. Mechanistic insights into the cytochrome P450-mediated oxidation and rearrangement of littorine in tropane alkaloid biosynthesis. Chem Bio Chem 10 , 2382-2393 (2009).

19. Matsuda, J., Okabe, S., Hashimoto, T. \& Yamada, Y. Molecular cloning of hyoscyamine 6 $\beta$-hydroxylase, a 2-oxoglutarate-dependent dioxygenase, from cultured roots of Hyoscyamus niger. J. Biol. Chem. 266, 9460-9464 (1991).

20. Hashimoto, T., Matsuda, J. \& Yamada, Y. Two-step epoxidation of hyoscyamine to scopolamine is catalyzed by bifunctional hyoscyamine $6 \beta$-hydroxylase. FEBS Lett. 329, 35-39 (1993).

21. Wang, Z., Jiang, M., Guo, X., Liu, Z. \& He, X. Reconstruction of metabolic module with improved promoter strength increases the productivity of 2-phenylethanol in Saccharomyces cerevisiae. Microb. Cell Fact. 17, 60 (2018).

22. Zhang, X., Zhang, S., Shi, Y., Shen, F. \& Wang, H. A new high phenyl lactic acid-yielding Lactobacillus plantarum IMAU10124 and a comparative analysis of lactate dehydrogenase gene. FEMS Microbiol. Lett. 356, 89-96 (2014).

23. Sévin, D. C., Fuhrer, T., Zamboni, N. \& Sauer, U. Nontargeted in vitro metabolomics for high-throughput identification of novel enzymes in Escherichia coli. Nat. Methods 14, 187-194 (2017).

24. Qiu, F. et al. A phenylpyruvic acid reductase is required for biosynthesis of tropane alkaloids. Org. Lett. 24, 7807-7810 (2018).

25. Fujii, T. et al. Novel fungal phenylpyruvate reductase belongs to D-isomer-specific 2-hydroxyacid dehydrogenase family. Biochim. Biophys. Acta. Proteins Proteomics 1814, 1669-1676 (2011)

26. Zheng, Z. et al. Efficient conversion of phenylpyruvic acid to phenyllactic acid by using whole cells of Bacillus coagulans SDM. PLoS ONE 6, e19030 (2011).

27. Li, J. F. et al. Directed modification of L-LCLDH1, an L-lactate dehydrogenase from Lactobacillus casei, to improve its specific activity and catalytic efficiency towards phenylpyruvic acid. J. Biotechnol. 281, 193-198 (2018).

28. Ross, J., Li, Y., Lim, E.-K. \& Bowles, D. J. Higher plant glycosyltransferases. Genome Biol. 2, 3004.1-3004.6 (2001).

29. Wang, H. et al. Engineering Saccharomyces cerevisiae with the deletion of endogenous glucosidases for the production of flavonoid glucosides. Microb. Cell Fact. 15, 134 (2016).

30. Michigan State University. Medicinal Plant Genomics Resource (accessed online 23 April 23, 2018); http://medicinalplantgenomics.msu.edu.

31. Grabherr, M. G. et al. Full-length transcriptome assembly from RNA-seq data without a reference genome. Nat. Biotechnol. 29, 644-652 (2011).

32. NIH. Medicinal Plant RNA Seq Database (accessed online 12 July 2018); https:// medplantrnaseq.org.

33. Bontpart, T., Cheynier, V., Ageorges, A. \& Terrier, N. BAHD or SCPL acyltransferase? What a dilemma for acylation in the world of plant phenolic compounds. New Phytol. 208, 695-707 (2015).

34. Carqueijeiro, I. et al. in Plant Vacuolar Trafficking. Methods and Protocols 33-54 (2018).

35. Strasser, R. Plant protein glycosylation. Glycobiology 26, 926-939 (2016).

36. Stehle, F., Stubbs, M. T., Strack, D. \& Milkowski, C. Heterologous expression of a serine carboxypeptidase-like acyltransferase and characterization of the kinetic mechanism. FEBS J. 275, 775-787 (2008).

37. Stack, J. H., Horazdovsky, B. \& Emr, S. D. Receptor-mediated protein sorting to the vacuole in yeast: roles for a protein kinase, a lipid kinase and GTP-binding proteins. Annu. Rev. Cell Dev. Biol. 11, 1-33 (1995).

38. Roberts, C. J., Nothwehr, S. F. \& Stevens, T. H. Membrane protein sorting in the yeast secretory pathway: evidence that the vacuole may be the default compartment. J. Cell Biol. 119, 69-83 (1992)

39. Liu, L., Spurrier, J., Butt, T. R. \& Strickler, J. E. Enhanced protein expression in the baculovirus/insect cell system using engineered SUMO fusions. Protein Expr. Purif. 62, 21-28 (2008).

40. Morita, M. et al. Vacuolar transport of nicotine is mediated by a multidrug and toxic compound extrusion (MATE) transporter in Nicotiana tabacum. Proc. Natl Acad. Sci. USA 106, 2447-2452 (2009)

41. Shoji, T. et al. Multidrug and toxic compound extrusion-type transporters implicated in vacuolar sequestration of nicotine in tobacco roots. Plant Physiol. 149, 708-718 (2009).

42. Cardillo, A. B., Perassolo, M., Sartuqui, M., Rodríguez Talou, J. \& Giulietti, A. M. Production of tropane alkaloids by biotransformation using recombinant Escherichia coli whole cells. Biochem. Eng. J. 125, 180-189 (2017).

43. Lesuisse, E., Crichton, R. R. \& Labbe, P. Iron-reductases in the yeast Saccharomyces cerevisiae. Protein Struct. Mol. 1038, 253-259 (1990)

44. Hu, Y., Zhu, Z., Nielsen, J. \& Siewers, V. Heterologous transporter expression for improved fatty alcohol secretion in yeast. Metab. Eng. 45, 51-58 (2018).

45. Dastmalchi, M. et al. Purine permease-type benzylisoquinoline alkaloid transporters in opium poppy. Plant Physiol. 181, 916-933 (2019).

46. Ro, D. K. et al. Induction of multiple pleiotropic drug resistance genes in yeast engineered to produce an increased level of anti-malarial drug precursor, artemisinic acid. BMC Biotechnol. 8, 83 (2008).

47. Brown, S., Clastre, M., Courdavault, V. \& O'Connor, S. E. De novo production of the plantderived alkaloid strictosidine in yeast. Proc. Natl Acad. Sci. USA 112, 3205-3210 (2015).

48. Galanie, S., Thodey, K., Trenchard, I. J., Filsinger Interrante, M. \& Smolke, C. D. Complete biosynthesis of opioids in yeast. Science 349, 1095-1100 (2015).

49. Cravens, A., Payne, J. \& Smolke, C. D. Synthetic biology strategies for microbial biosynthesis of plant natural products. Nat. Commun. 10, 2142 (2019).

50. Redford, K. H., Brooks, T. M., Macfarlane, N. B. W. \& Adams, J. S. Genetic Frontiers for Conservation: an Assessment of Synthetic Biology and Biodiversity Conservation https:// portals.iucn.org/library/node/48408 (2019).

Publisher's note Springer Nature remains neutral with regard to jurisdictional claims in published maps and institutional affiliations.

(c) The Author(s), under exclusive licence to Springer Nature Limited 2020 


\section{Methods}

\section{Data reporting}

No statistical methods were used to predetermine sample size. The experiments were not randomized and investigators were not blinded to allocation during experiments and outcome assessment.

\section{Chemical compounds and standards}

Tropine, $(S)$-hyoscyamine hydrobromide and $(S)$-scopolamine hydrobromide were purchased from Santa Cruz Biotechnology. $(R)$-Littorine hydrochloride was purchased from Toronto Research Chemicals. All other chemicals were purchased from Sigma.

\section{Plasmid construction}

DNA oligonucleotides used in this study were synthesized by the Stanford Protein and Nucleic Acid Facility and are listed in Supplementary Data 1 . Genes encoding biosynthetic enzymes used in this study are listed by source and accession number in Supplementary Table 2; for $H D H$ genes newly identified in this work, full amino acid sequences are given in Supplementary Table 1. Endogenous yeast genes were amplified from Saccharomyces cerevisiae CEN.PK2-1D ${ }^{51}$ genomic DNA via colony $\mathrm{PCR}^{52}$. Gene sequences encoding heterologous enzymes were codon-optimized for expression in S. cerevisiae using GeneArt GeneOptimizer software (Thermo Fisher Scientific) and synthesized as double-stranded gene fragments (Twist Bioscience). Plasmids used in this study are listed in Supplementary Data 2. Three types of plasmids were used in this work: yeast expression plasmids, yeast integration plasmids, and Agrobacterium tumefasciens binary vectors.

Yeast expression plasmids harboured a gene of interest flanked by a constitutive promoter and terminator, an auxotrophic selection marker, and either a low-copy CEN6/ARS4 or a high-copy $2 \mu$ yeast origin of replication. These plasmids were constructed by addition of $5^{\prime}$ and 3 ' restriction sites to genes of interest using PCR, restriction digestion of PCR amplicons or synthesized gene fragments, and ligation of digested inserts into similarly digested vectors pAG414GPD-ccdB, pAG415GPD-ccdB, pAG416GPD-ccdB, pAG424GPD-ccdB, pAG425GPD-ccdB or pAG426GPD-ccdB ${ }^{53}$ using T4 DNA ligase (New England Biolabs, NEB). Yeast expression plasmids expressing fusions of multiple proteins or enzymes were prepared by PCR amplification of each gene of interest with $15-25$ bp of overlap to adjacent fragments, assembly of fragments into single inserts with $5^{\prime}$ and $3^{\prime}$ restriction sites using overlap-extension PCR, and ligation cloning into digested vectors as described.

Yeast integration plasmids comprised a gene of interest flanked by a constitutive promoter and terminator, but lacked a selection marker and origin of replication for yeast expression. These plasmids were constructed by PCR linearization of the empty holding vectors pCS2656, pCS2657, pCS2658, pCS2661 or pCS2663 using primers complementary to the $3^{\prime}$ and $5^{\prime}$ ends of the promoter and terminator, respectively. Genes intended for yeast genomic integration were PCR-amplified to append 5' and 3' overhangs with 35-40 bp of homology to the termini of the linearized holding vectors and then assembled using Gibson assembly.

For transient expression of littorine synthase variants in Nicotiana benthamiana, A. tumefasciens binary vectors contained a transfer-DNA (T-DNA) region comprising a gene of interest flanked by the constitutive Cauliflower Mosaic Virus (CaMV) 35S promoter/Cowpea Mosaic Virus (CPMV) 5'UTR and a nopaline synthase terminator, as well as an analogous expression cassette for the p19 RNAi-suppressor protein. These plasmids were constructed via addition of 5' Agel and $3^{\prime}$ Xhol restriction sites to a gene of interest via PCR, followed by digestion and ligation into the $\mathrm{pEAQ}-\mathrm{HT}$ binary vector $\mathrm{pCS} 3352^{54}$.

All PCR amplification was performed using Q5DNA polymerase (NEB) and linear DNA was purified using the DNA Clean and Concentrator-5 kit (Zymo Research). Assembled plasmids were transformed into chemically competent $E$. coli (TOP10, Thermo Fisher Scientific) via heat-shock and propagated with selection in Luria-Bertani (LB) broth or on LB-agar plates with either carbenicillin $\left(100 \mu \mathrm{g} \mathrm{ml}^{-1}\right)$ or kanamycin $\left(50 \mu \mathrm{g} \mathrm{ml}^{-1}\right)$ selection. E. coli plasmid DNA was isolated by alkaline lysis from overnight cultures grown at $37^{\circ} \mathrm{C}$ and $250 \mathrm{rpm}$ in selective LB media using Econospin columns (Epoch Life Science) according to the manufacturer's protocol. Plasmid sequences were verified by Sanger sequencing (Quintara Biosciences).

\section{Yeast strain construction}

Yeast strains used in this study (Supplementary Table 3) were derived from our previously reported tropine-producing strain $\mathrm{CSY} 1251^{14}$, which is in turn derived from the parental strain CEN.PK2-1D ${ }^{51}$. Strains were grown non-selectively in yeast-peptone media supplemented with $2 \%$ $\mathrm{w} / \mathrm{v}$ dextrose (YPD media), yeast nitrogen base (YNB) defined media (Becton, Dickinson and Company, BD) supplemented with synthetic complete amino acid mixture (YNB-SC; Clontech) and 2\% (w/v) dextrose, or on agar plates of the aforementioned media. Strains transformed with plasmids bearing auxotrophic selection markers (URA3, $T R P 1$ and/or $L E U 2$ ) were grown selectively in YNB media supplemented with $2 \% \mathrm{w} / \mathrm{v}$ dextrose and the appropriate dropout solution (YNB-DO; Clontech) or on YNB-DO agar plates.

Yeast genomic modifications were performed using the CRISPRm method $^{55}$. CRISPRm plasmids expressing Streptococcuspyogenes Cas 9 and a single guide RNA (sgRNA) targeting a genomic locus were constructed by assembly PCR and Gibson assembly of DNA fragments encoding SpCas9 (pCS3410), tRNA promoter and HDV ribozyme (pCS3411), a 20-nucleotide guide RNA sequence oligonucleotide, and tracrRNA and terminator (pCS3414) (Supplementary Data 2). For gene insertions, integration fragments comprising one or more genes of interest flanked by unique promoters and terminators were PCR-amplified from yeast integration plasmids using Q5 DNA polymerase (NEB) with flanking $40 \mathrm{bp}$ microhomology regions to adjacent fragments and/or to the yeast genome at the integration site (Extended Data Fig. 1, Supplementary Data 1). For gene disruptions, integration fragments comprised 6-8 stop codons in all three reading frames flanked by $40 \mathrm{bp}$ of microhomology to the disruption site, which was located within the first half of the open reading frame. Approximately 0.5-1 $\mu \mathrm{g}$ of each integration fragment was co-transformed with $500 \mathrm{ng}$ of multiplex CRISPR plasmid targeting the desired genomic site. Positive integrants were identified by yeast colony $\mathrm{PCR}^{52}$, Sanger sequencing, and/or functional screening by LC-MS/MS.

\section{Yeast transformations}

Yeast strains were chemically transformed using the Frozen-EZ Yeast Transformation II Kit (Zymo Research) as per the manufacturer's instructions, with the following modifications. For competent cell preparation, individual colonies were inoculated into YPD media and grown overnight at $30^{\circ} \mathrm{C}$ and $460 \mathrm{rpm}$. Saturated cultures $(-14-18 \mathrm{~h})$ were back-diluted between 1:10 and 1:50 in fresh YPD media and grown to exponential phase $(\sim 5-7 \mathrm{~h})$. Cultures were pelleted by centrifugation at $500 \mathrm{~g}$ for $4 \mathrm{~min}$, washed twice with $50 \mathrm{mM}$ Tris-HCl buffer ( $\mathrm{pH} \mathrm{8.5),}$ and then resuspended in 20-50 $\mu \mathrm{l}$ of EZ2 solution per transformation. For transformation, competent cells were mixed with 250-1,000 ng of total DNA and 200-500 $\mu \mathrm{l}$ of EZ3 solution. Cell suspensions were incubated at $30^{\circ} \mathrm{C}$ with slow rotation for $1-1.5 \mathrm{~h}$. For plasmid transformations, the transformed yeast were directly plated onto YNB-DO agar plates. For CRISPRm genomic modifications, yeast suspensions were instead mixed with $1 \mathrm{ml} \mathrm{YPD}$ media, pelleted by centrifugation at $500 \mathrm{~g}$ for $4 \mathrm{~min}$, and then resuspended in 300-500 $\mu \mathrm{l}$ of fresh YPD medium. Suspensions were incubated at $30^{\circ} \mathrm{C}$ with gentle rotation for $2-3 \mathrm{~h}$ to allow expression of geneticin resistance and then spread on YPD plates supplemented with $200-400 \mathrm{mg} \mathrm{l}^{-1} \mathrm{G} 418$ (geneticin) sulfate. Plates were incubated at $30^{\circ} \mathrm{C}$ for $72 \mathrm{~h}$ to allow sufficient colony formation before downstream applications. 


\section{Growth conditions for metabolite assays}

Small-scale metabolite production assays were performed in YNB-SC or YNB-DO media supplemented with $2 \%$ dextrose and $5 \%$ glycerol (YNB-G) for optimal tropine production ${ }^{14}$ in at least three replicates. Our previous work showed that tropine biosynthesis is significantly enhanced by higher starting cell densities ${ }^{14}$. Therefore, yeast colonies were initially inoculated in triplicate into $1 \mathrm{ml} \mathrm{YPD} \mathrm{or} \mathrm{YNB-DO} \mathrm{and} \mathrm{grown}$ to saturation $(-18-22 \mathrm{~h})$ at $30^{\circ} \mathrm{C}$ and $460 \mathrm{rpm}$, pelleted by centrifugation at $500 \mathrm{~g}$ for $4 \mathrm{~min}$ and 3,000 $\mathrm{g}$ for $1 \mathrm{~min}$, resuspended in $1 \mathrm{ml}$ of fresh selective or non-selective YNB-G media (for some experiments, additionally supplemented with $15 \mathrm{mg} \mathrm{l}^{-1} \mathrm{Fe}^{2+}$ from iron (II) sulfate and $50 \mathrm{mM}$ 2-oxoglutarate ${ }^{56}$ ), and then $300 \mu \mathrm{l}$ transferred into $2 \mathrm{ml}$ deep-well 96-well plates sealed with AeraSeal gas-permeable film (Excel Scientific). Cultures were grown for $72-96 \mathrm{~h}$ at $25^{\circ} \mathrm{C}, 460 \mathrm{rpm}$, and $80 \%$ relative humidity in a Lab-Therm LX-T shaker (Adolf Kuhner).

\section{Growth conditions for time courses}

To simulate high-density batch culture conditions, strains were inoculated in triplicate into $10 \mathrm{ml}$ of YPD media or selective YNB-G media and grown overnight to saturation at $30^{\circ} \mathrm{C}$ and $250 \mathrm{rpm}$. Saturated cultures were pelleted by centrifugation at $500 \mathrm{~g}$ for $4 \mathrm{~min}$ and $3,000 \mathrm{~g}$ for $1 \mathrm{~min}$ and then resuspended in $10 \mathrm{ml}$ of fresh selective or non-selective YNB-G media supplemented with $50 \mathrm{mM}$ 2-oxoglutarate and $15 \mathrm{mg}$ $\mathrm{I}^{-1} \mathrm{Fe}^{2+}$, and grown in $50-\mathrm{ml}$ shake flasks with $10 \mathrm{ml}$ starting volume in triplicates at $25^{\circ} \mathrm{C}$ and $300 \mathrm{rpm}$ for $120 \mathrm{~h}$. Where indicated, fed-batch conditions were approximated by supplementing cultures after $72 \mathrm{~h}$ of growth with appropriate carbon sources and amino acids at $2 \%$ and $1 \times$ final concentrations, respectively. At appropriate time points, $250 \mu \mathrm{l}$ samples were removed from cultures for analysis; $100 \mu \mathrm{l}$ of culture was diluted $10 \times$ and used for optical density measurement at $600 \mathrm{~nm}$ on a Nanodrop 2000c spectrophotometer, and $150 \mu \mathrm{l}$ of culture was used for metabolite quantification.

\section{Analysis of metabolite production}

Yeast cultures were pelleted by centrifugation at 3,500 $\mathrm{g}$ for $5 \mathrm{~min}$ at $12^{\circ} \mathrm{C}$ and $150 \mu$ l aliquots of supernatant were removed for analysis. Metabolites were analysed by LC-MS/MS using an Agilent 1260 Infinity Binary HPLC and an Agilent 6420 Triple Quadrupole mass spectrometer. Chromatography was performed using a Zorbax EclipsePlus C18 column $(2.1 \times 50 \mathrm{~mm}, 1.8 \mu \mathrm{m}$; Agilent Technologies $)$ with $0.1 \%(\mathrm{v} / \mathrm{v})$ formic acid in water as mobile phase solvent $A$ and $0.1 \%(v / v)$ formic acid in acetonitrile as solvent $B$. The column was operated with a constant flow rate of $0.4 \mathrm{ml} \mathrm{min}^{-1}$ at $40^{\circ} \mathrm{C}$ and a sample injection volume of $10 \mu \mathrm{l}$. Chromatographic separation was performed using the following gradient $^{14}$ : 0.00-0.75 min, $1 \% \mathrm{~B} ; 0.75-1.33 \mathrm{~min}, 1-25 \% \mathrm{~B} ; 1.33-2.70 \mathrm{~min}$, 25-40\% B; 2.70-3.70 min, 40-60\% B; 3.70-3.71 min, 60-95\% B; 3.71-4.33 min, $95 \%$; $4.33-4.34 \mathrm{~min}, 95-1 \% \mathrm{~B} ; 4.34-5.00 \mathrm{~min}$, equilibration with $1 \%$ B. For separation and detection of phenylpropanoid acyl donors (PLA, cinnamate and ferulate) and corresponding glucosides, the final equilibration step at $1 \%$ B was extended to $4.34-7.50 \mathrm{~min}$. The LC eluent was directed to the MS from 0.01-5.00 min operating with electrospray ionization (ESI) in positive mode, source gas temperature $350^{\circ} \mathrm{C}$, gas flow rate $11 \mathrm{I} \mathrm{min}^{-1}$, and nebulizer pressure 40 psi. Data collection was performed using MassHunter Workstation LC/MS Data Acquisition software (Agilent). Metabolites were identified and quantified by integrated peak area in MassHunter Workstation Qualitative Analysis Navigator software (Agilent) using the mass fragment/transition parameters in Supplementary Table 4 and standard curves. Primary MRM transitions were identified by analysis of 0.1-1 $\mathrm{mM}$ aqueous standards using MassHunter Workstation Optimizer software (Agilent) and corroborated against published mass transitions if available, and/or against predicted transitions determined using the CFM-ID fragment prediction utility ${ }^{57}$ and the METLIN database ${ }^{58}$. As both PLA and its glucoside formed strong ammonium adducts, these metabolites were detected and quantified in positive mode using the corresponding $[\mathrm{M}+\mathrm{H}+17]^{+}$ions, $m / z 184$ (PLA) and 346 (PLAglucoside) (Supplementary Table 4).

\section{Fluorescence microscopy}

Individual colonies of yeast strains transformed with plasmids encoding biosynthetic enzymes fused to fluorescent protein reporters were inoculated into $1 \mathrm{ml}$ selective or non-selective YNB-G media and grown overnight $(-14-18 \mathrm{~h})$ at $30^{\circ} \mathrm{C}$ and $460 \mathrm{rpm}$. Overnight cultures were back-diluted between 1:2 and 1:4 into fresh YNB-G media and grown to exponential phase at $30^{\circ} \mathrm{C}$ and $460 \mathrm{rpm}$ for an additional $6-8 \mathrm{~h}$ to allow slow-maturing fluorescent proteins to fold before imaging.

Yeast vacuoles were co-imaged with fluorescent reporter-fused biosynthetic enzymes using the FM4-64 stain (Thermo Fisher) and pulse-chase fluorescence microscopy. FM4-64 is a red-fluorescent lipophilic styryl dye that intercalates into the yeast plasma membrane and is endocytosed during growth on rich media, accumulating in vacuolar membranes ${ }^{59}$. Transformed yeast colonies were inoculated into $1 \mathrm{ml}$ selective or non-selective YNB-G and grown overnight ( 14-18 h) at $30^{\circ} \mathrm{C}$ and $460 \mathrm{rpm}$, then back-diluted between $1: 10$ and 1:3 into $1 \mathrm{ml}$ of fresh YNB-G and grown for an additional $2-4 \mathrm{~h}$ until $\mathrm{OD}_{600}$ value of $0.5-0.8$. Cultures were pelleted by centrifugation at $5,000 \mathrm{~g}$ for 5

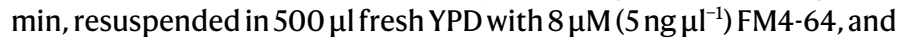
incubated at $30^{\circ} \mathrm{C}$ for $30 \mathrm{~min}$ with gentle rotation. Stained cells were pelleted by centrifugation at 3,000g for $5 \mathrm{~min}$ (pellets were visibly red), washed twice with $1 \mathrm{ml}$ YPD, resuspended in $5 \mathrm{ml} \mathrm{YPD}$, and then incubated at $30^{\circ} \mathrm{C}$ and $460 \mathrm{rpm}$ for $90-120 \mathrm{~min}$ to allow endocytosis and vacuolar accumulation of the dye. Cultures were pelleted by centrifugation at $500 \mathrm{~g}$ for $4 \mathrm{~min}$ followed by $3,000 \mathrm{~g}$ for $1 \mathrm{~min}$, then resuspended in $250 \mu$ lof $40 \mathrm{mM}$ MES buffer ( $\mathrm{pH} 6.5$ ) and imaged immediately.

For imaging, approximately $5-10 \mu$ of cell suspension was spotted onto a glass microscope slide and covered with a glass coverslip (Thermo Fisher) and then imaged using an upright Zeiss Axiolmager Epifluorescence/Widefield microscope with a $\times 64$ oil immersion objective. Fluorescence excitation was performed using an EXFOX-Cite 120 illumination source and the following Semrock Brightline filter settings: GFP, 472/30 excitation and 520/35 emission; mCherry/DsRed/Cy3/ TexasRed, 562/40 excitation and 624/40 emission. Emitted light was captured with a Zeiss Axiocam 503 mono camera and Zen Pro software, and subsequent image analysis was performed in ImageJ/Fiji (NIH). Images were converted to pseudocolor using the 'merge channels' and 'split channels' functions (Image $\rightarrow$ Colour $\rightarrow$ Merge/Split Channels). For each sample, linear histogram stretching was applied across all images for a given channel to improve contrast.

To reduce the interference of light from other focal planes when imaging sub-cellular organelles, we performed 2D digital deconvolution analysis, a common computational technique used for removing out-of-focus light distortion from 2D images of 3D structures ${ }^{60}$. First, a theoretical point-spread function (PSF), which mathematically describes the diffraction of light from a point source in a specific imaging setup, was computed using the 'Diffraction PSF 3D' plugin for ImageJ (available from http://fiji.sc/Diffraction_PSF_3D) for the green and red channels using the following parameters: index of refraction of the media, 1.518 (lens oil); numerical aperture, 1.40 ; wavelength (nm), 520 (green) or 624 (red); longitudinal spherical aberration at max. aperture (nm), 0.00 (default); image pixel spacing $(\mathrm{nm}), 72$; slice spacing $(\mathrm{nm}), 0$; width (pixels), 240; height (pixels), 242; depth (slices), 1; normalization, sum of pixel values $=1$. Next, green and red channel images were separately deconvolved against the corresponding PSFs using the 'Parallel Spectral Deconvolution 2D' plugin for ImageJ (available from http://fiji.sc/Parallel_ Spectral_Deconvolution) with default settings and auto regularization.

\section{Identification of HDH candidates}

Tissue-specific abundances (fragments per kilobase of contig per million mapped reads, FPKM) and putative protein structural and 
functional annotations for each of 43,861 unique transcripts identified from the $A$. belladonna transcriptome were obtained from the MSU Medicinal Plant Genomics Resource ${ }^{30}$. Transcripts encoding hyoscyamine dehydrogenase candidates were identified based on clustering of tissue-specific expression profiles with those of the bait genes CYP8OF1 (littorine mutase) and $H 6 H$ (hyoscyamine $6 \beta$-hydroxylase/ dioxygenase), which respectively precede and follow the dehydrogenase step in the TA biosynthetic pathway, using a custom R script which is described below.

First, the complete list of 43,861 transcripts was filtered for those annotated with any of the following oxidoreductase protein family (PFAM) IDs: PF00106,PF13561,PF08659, PF08240, PF00107, PF00248, PF00465, PF13685, PF13823, PF13602, PF16884 and PF00248; or any of the following functional annotation keywords: alcohol dehydrogenase, aldehyde reductase, short chain, aldo/keto. In addition, any transcripts with functional annotations containing the keywords putrescine, tropinone and tropine were included in the filter as positive control TA-associated genes to validate clustering with bait genes. Next, mean tissue-specific expression profiles were generated for the CYP80F1 and H6H bait genes. For each of the two bait genes, linear regression models were constructed to express the bait gene expression profile (in FPKM) as a linear function of each candidate gene profile and correlation $P$ values were computed for each candidate. The candidates identified using each of the two bait genes were pooled and duplicates were removed. Combined $P$ values for each candidate were computed as the sum of the $\log _{10}(P$ values $)$ of the correlations with each of the two bait genes. Transcripts matching known dehydrogenases in the TAbiosynthetic pathway (that is, tropinone reductases I and II) were removed, and the remaining candidates were ranked by combined $P$ value and by distance from bait genes via hierarchical clustering of tissue-specific expression profiles.

\section{De novo transcriptome assembly}

All data pre-processing, analysis, and de novo transcriptome assembly was performed on the Sherlock2.0 high-performance computing cluster (HPCC) at Stanford University. Paired-end raw RNA-seq reads corresponding to $A$. belladonna secondary roots (accession SRX060267, run SRR192881) and sterile seedling tissue (accession SRX060269, run SRR192882) were retrieved from the NCBI Sequence Read Archive (SRA) using the SRA Toolkit (NIH). The paired-end raw reads were analysed via FastQC (Babraham Bioinformatics) and then trimmed using the BBDuk. sh utility (Joint Genome Institute, Department of Energy) using the following parameters: $k$-mer trimming, right end only ('ktrim=r'); $k$-mer length, 23 (' $\mathrm{k}=23$ '); minimum k-mer for end-trimming, 11 ('mink=11'), Hamming distance for $\mathrm{k}$-mer matching, 1 ('hdist $=\mathbf{1}^{\prime}$ ); trim paired reads to same length ('tpe'), trim adapters using pair overlap detection ('tbo'); quality trimming, both right and left ends ('qtrim=rl'); quality cut-off, 5 ('trimq $=5$ '); minimum permissible read length after trimming, 25 ('minlen $=25$ '). Two independent de novo transcriptome assemblies were generated from the processed paired-end reads from secondary root (SRR192881) and seedling (SRR192882), respectively, using the Trinity software suite with default settings ${ }^{31,61}$.

Transcript functional annotation for each of the two assemblies (secondary root and seedling) was performed using the Trinotate package ${ }^{62}$. Following coding region prediction using the TransDecoder.LongOrfs and TransDecoder.Predict commands, annotations were generated using a BLASTp search against the UniProt/SwissProt databases and a protein homology search using HMMER. Complete ORF sequences for each of the candidate transcripts identified from co-expression analysis were generated by performing tBLASTn and tBLASTx searches against the Trinity transcriptome assemblies; hits with protein percent identity of at least $98 \%$, accounting for sequencing errors, were assumed to be identical.

\section{Identification of orthologues from transcriptome databases}

Orthologues of $A$. belladonna UGT84A27 (UGT) were identified using tBLASTn searches of the transcriptomes of Brugmansia sanguinea and
Datura metel in the 1000Plants (1KP) database ${ }^{63}$. This search yielded two unique, full-length amino acid sequences (that is, within $5 \%$ of the length of the query sequence) and with expectation value 0.0: scaffold-AIOU-2012986-Brugmansia_sanguinea (B. sanguinea,BsUGT) and scaffold-JNVS-2051323-Datura_metel (D. metel,DmUGT).

Orthologues of HDH were identified using tBLASTn searches of the transcriptomes of several Datura species in the Medicinal Plant RNA-seq database ${ }^{32}$. This search yielded two unique, full-length amino acid sequences (that is, within $5 \%$ of the length of the query sequence) and with expectation value 0.0: medp_datin_20101112|6354 (DiHDH) and medp_datst_20101112|10433 (DsHDH).

Coding sequences for all putative orthologues were optimized for yeast expression and then cloned into expression vectors as described in 'Plasmid construction'.

\section{Protein structural analysis and substrate docking}

Homology models of $A b \mathrm{UGT}, A b \mathrm{HDH}$ and $A b \mathrm{LS}$ were constructed using RaptorX with default modelling parameters ${ }^{64}$. For docking simulations, the binding of cosubstrates (UDP-glucose for $A b U G T$ ) or cofactors (NADPH for $A b \mathrm{HDH}$ ) was first predicted based on structural alignment with the crystal structures of $A$. thaliana salicylate UDP-glucosyltransferase UGT74F2 with bound UDP (PDB: 5V2K) and Populus tremuloides sinapyl alcohol dehydrogenase with bound NADPH (PDB: 1YQD) respectively, as the binding pockets for these cosubstrates are tightly conserved. Geometry optimizations of substrate structures (PLA or hyoscyamine aldehyde) before docking simulations were conducted using the Gaussian 16 software package on the Stanford Sherlock2.0 HPCC (run parameters: DFT, B3LYP, LANL2DZ). The energy-minimized ligand structures were then docked into the corresponding cosubstrate/cofactor-bound homology models using the Maestro and Glide XP software packages (Schrodinger) with default parameters; for the docking of hyoscyamine aldehyde into $A b \mathrm{HDH}$, a spatial constraint of maximum $6 \AA$ separation between the aldehyde carbon and the catalytic $\mathrm{Zn}^{2+}$ was additionally imposed ${ }^{65}$. Enzyme structures and docking results were visualized using PyMOL software (Schrodinger).

\section{Phylogenetic analysis of HDH orthologues}

Phylogenetic tree construction was based on a BLASTp search using $A b \mathrm{HDH}$ as a query against the UniProt/SwissProt database (annotated sequences only). Sequences chosen for tree construction included the top 50 BLASTp hits based on $E$-value, as well as 10 additional hits selected from among the next 100 ranks. Phylogenetic relationships were derived via bootstrap neighbour-joining with $n=1,000$ trials in ClustalX2 and the resulting tree was visualized with FigTree software.

\section{Expression of littorine synthase HA-tagged variants in tobacco}

Binary vector (pEAQ-HT-based) plasmids were transformed into A. tumefasciens (GV3101) using the freeze-thaw method ${ }^{66}$. Transformants were grown on LB-agar plates supplemented with $50 \mu \mathrm{g} \mathrm{ml}^{-1}$ kanamycin and $30 \mu \mathrm{g} \mathrm{ml}^{-1}$ gentamicin at $30^{\circ} \mathrm{C}$ for $48 \mathrm{~h}$. Colonies were inoculated

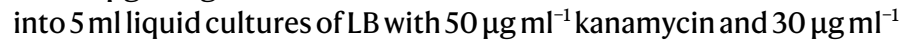
gentamicin and grown for $18-24 \mathrm{~h}$ at $30^{\circ} \mathrm{C}$ and $250 \mathrm{rpm}$ in a shaking incubator. Saturated cultures were pelleted by centrifugation at $5,000 \mathrm{~g}$ for $5 \mathrm{~min}$. Pellets were resuspended in the same volume $(-5 \mathrm{ml})$ of freshly prepared infiltration buffer (10 $\mathrm{mM} \mathrm{MES} \mathrm{buffer,} \mathrm{pH}$ 5.6, $10 \mathrm{mM} \mathrm{MgCl}_{2}$, $150 \mu \mathrm{M}$ acetosyringone), incubated at room temperature for $2-3 \mathrm{~h}$ with gentle rocking to prevent settling, and then diluted in infiltration buffer to $\mathrm{OD}_{600}$ of $0.8-1.0$. N. benthamiana plants were grown for 4 weeks under a $16 \mathrm{~h} \mathrm{light} / 8 \mathrm{~h}$ dark cycle before infiltration. Approximately $1-2 \mathrm{ml}$ of Agrobacterium cell suspension was infiltrated into the underside of each of the three largest leaves of each plant using a needleless $1 \mathrm{ml}$ syringe. Leaves were harvested four days after infiltration, flash-frozen in liquid nitrogen, and stored at $-80^{\circ} \mathrm{C}$ for downstream processing. 
All infiltrations were performed in triplicate, in which one biological replicate comprised three infiltrated leaves from a single plant.

\section{Deglycosylation of yeast- and tobacco-expressed littorine synthase}

Removal of $\mathrm{N}$ - and $\mathrm{O}$-linked glycosylation from littorine synthase in yeast and $N$. benthamiana crude cell lysate was performed using PNGase F and O-glycosidase (NEB), respectively, following the manufacturer's protocols. In brief, approximately $30 \mu \mathrm{g}$ of total protein containing LS in crude cell lysate was denatured in $1 \times$ glycoprotein denaturing buffer at $100^{\circ} \mathrm{C}$ for $10 \mathrm{~min}$, followed by immediate chilling on ice. Denatured lysates were deglycosylated using $\mathrm{PNGase} \mathrm{F}$ or O-glycosidase as per manufacturer instructions at $37^{\circ} \mathrm{C}$ for $1 \mathrm{~h}$, then stored at $-20^{\circ} \mathrm{C}$ until analysis.

\section{Analysis of protein expression by western blot}

For immunoblot analysis of yeast-expressed proteins, strain CSY1294 was transformed with HA-tagged AbLS expression vectors as described in 'Yeast transformations'. Three days after transformation, transformed colonies were inoculated into $2 \mathrm{ml} \mathrm{YNB-DO}$ media and grown overnight $(-16-20 \mathrm{~h})$ to stationary phase at $30^{\circ} \mathrm{C}$ and $460 \mathrm{rpm}$. Cells were pelleted by centrifugation at $3,000 \mathrm{~g}$ for $5 \mathrm{~min}$, resuspended in $200 \mu \mathrm{H} \mathrm{H}_{2} \mathrm{O}$, mixed with $200 \mu \mathrm{l}$ of $0.2 \mathrm{M} \mathrm{NaOH}$, and incubated at room temperature for $5 \mathrm{~min}$ to allow hydrolysis of cell wall glycoproteins ${ }^{67}$. Cells were re-pelleted at 3,000g for $5 \mathrm{~min}$, resuspended in $75 \mu \mathrm{H}_{2} \mathrm{O}$, mixed with $25 \mu$ l of $4 \times$ NuPAGE LDS sample buffer (Thermo Fisher), and then boiled at $95^{\circ} \mathrm{C}$ for $3 \mathrm{~min}$ to lyse cells. Suspensions were pelleted by centrifugation at $16,000 \mathrm{~g}$ for $5 \mathrm{~min}$ to remove insoluble debris and supernatants were transferred to pre-chilled tubes. Samples were stored at $-20^{\circ} \mathrm{C}$ until further analysis.

For analysis of tobacco-expressed proteins, all three infiltrated leaves from a single plant were ground together to a fine powder under liquid nitrogen and resuspended in $4-5 \mathrm{ml}$ of $25 \mathrm{mM}$ potassium phosphate buffer ( $\mathrm{pH} 8.0$ ) with HALT protease inhibitor cocktail (Thermo Fisher). Leaf homogenate slurries (final volume $7-8 \mathrm{ml}$ ) were incubated at $4{ }^{\circ} \mathrm{C}$ with gentle rotation for 45-60 min and then clarified by centrifugation at $9,000 \mathrm{~g}$ for $10 \mathrm{~min}$. Supernatant fractions were transferred to new tubes and re-clarified. Lysate protein concentrations were estimated using the Bio-RAD Protein Assay kit. Samples were stored at $-80^{\circ} \mathrm{C}$ until further analysis.

For analysis under reducing conditions, protein lysates were mixed with $\beta$-mercaptoethanol (final concentration 10\%) and incubated at $70{ }^{\circ} \mathrm{C}$ for $10 \mathrm{~min}$. Approximately $20-40 \mu \mathrm{g}$ of total protein was loaded onto NuPAGE Bis-Tris 4-12\% acrylamide gels (Thermo Fisher) with Precision Plus Dual Colour protein molecular mass marker (BioRad). Electrophoresis was conducted in $1 \times$ NuPAGE MOPS SDS running buffer at $150 \mathrm{~V}$ for $90 \mathrm{~min}$. Transfer of protein to nitrocellulose membranes was performed at $15 \mathrm{~V}$ for $15 \mathrm{~min}$ using a Trans-Blot Semi-Dry apparatus (BioRad) and NuPAGE transfer buffer (Thermo Fisher) per manufacturer instructions. For reducing conditions, NuPAGE antioxidant (Thermo Fisher) was added to a final concentration of $1 \times$ to both the running buffer and transfer buffer. Membranes with transferred protein were washed for $5 \mathrm{~min}$ in Tris-buffered saline with Tween (TBS-T; $137 \mathrm{mM} \mathrm{NaCl}, 2.7 \mathrm{mM} \mathrm{KCl}, 19 \mathrm{mM}$ Tris base, 0.1\% Tween20, pH 7.4) and then blocked with $5 \%$ skim milk in TBS-T for $1 \mathrm{~h}$ at room temperature. Membranes were incubated overnight at $4{ }^{\circ} \mathrm{C}$ with a 1:1,500 dilution of chimaeric rabbit IgGK anti-HA HRP-conjugated antibody (Absolute Antibody, 16.43/Ab00828-23.0) in TBS-T with 5\% milk, washed three times for 5 min each with TBS-T, and then visualized using Western Pico PLUS HRP substrate (Thermo Fisher) and a G:BOX gel imager (Syngene).

\section{Statistics}

Where indicated, the statistical significance of any differences in metabolite titer between conditions was verified using Student's two-tailed $t$-test in Microsoft Excel Professional 2013. For yeast experiments, biological replicates are defined as independent cultures inoculated from separate yeast colonies or streaks and cultivated in separate containers. For tobacco experiments, one biological replicate is defined as all infiltrated leaves from a single plant.

\section{Additional software}

All figures were prepared using GraphPad Prism 7, ImageJ, PyMOL, and Inkscape.

\section{Reporting summary}

Further information on research design is available in the Nature Research Reporting Summary linked to this paper.

\section{Data availability}

Data supporting the findings of this work are available within the paper and its Supplementary Information files. The datasets generated and analysed during the current study are available from the corresponding author upon reasonable request. Novel genetic sequences identified and characterized in this study are available from the following public databases. 1000Plants (1KP) database ${ }^{63}$ : scaffold-AIOU-20129 86-Brugmansia_sanguinea (BsUGT); scaffold-JNVS-2051323-Datura metel (DmUGT). Medicinal Plant RNA-seq database ${ }^{32}$ : medp_ $_{-}$ datin_20101112|6354 (DiHDH); medp_datst_20101112|10433 (DsHDH). MSU Medicinal Plant Genomics Resource ${ }^{30}$ : full amino acid sequences and database accession numbers (IDs) for all tested HDH candidates are provided in Supplementary Table 1 . Accession numbers for previously reported gene and protein sequences in the GenBank/UniProt databases are provided in Supplementary Table 2. Protein crystal structures used for homology modelling are available from the RCSB Protein Data Bank (PDB) with the following accessions: Arabidopsis thaliana salicylate UDP-glucosyltransferase UGT74F2 with bound UDP, accession 5V2K; P. tremuloides sinapyl alcohol dehydrogenase with bound $\mathrm{NADPH}$, accession 1YQD. Source data are provided with this paper.

\section{Code availability}

The custom R script used for identification of HDH candidates via coexpression analysis of $A$. belladonna RNA sequencing data is available from the Smolke Laboratory GitHub: https://github.com/smolkelab/ Oxidoreductase_coexpression_analysis.

51. Entian, K. D. \& Kötter, P. 25 yeast genetic strain and plasmid collections. Methods Microbiol. 36, 629-666 (2007).

52. Kwiatkowski, T. J., Jr, Zoghbi, H. Y., Ledbetter, S. A., Ellison, K. A. \& Chinault, A. C. Rapid identification of yeast artificial chromosome clones by matrix pooling and crude lysate PCR. Nucleic Acids Res. 18, 7191-7192 (1990).

53. Alberti, S., Gitler, A. D. \& Lindquist, S. A suite of Gateway cloning vectors for high-throughput genetic analysis in Saccharomyces cerevisiae. Yeast 24, 913-919 (2007).

54. Sainsbury, F., Thuenemann, E. C. \& Lomonossoff, G. P. pEAQ: versatile expression vectors for easy and quick transient expression of heterologous proteins in plants. Plant Biotechnol. J. 7, 682-693 (2009).

55. Ryan, O. W. et al. Selection of chromosomal DNA libraries using a multiplex CRISPR system. eLife 3, 1-15 (2014).

56. Thodey, K., Galanie, S. \& Smolke, C. D. A microbial biomanufacturing platform for natural and semisynthetic opioids. Nat. Chem. Biol. 10, 837-844 (2014).

57. Allen, F., Pon, A., Wilson, M., Greiner, R. \& Wishart, D. CFM-ID: a web server for annotation, spectrum prediction and metabolite identification from tandem mass spectra. Nucleic Acids Res. 42, W94-W99 (2014).

58. Guijas, C. et al. METLIN: a technology platform for identifying knowns and unknowns. Anal. Chem. 90, 3156-3164 (2018).

59. Amberg, D. C., Burke, D. J. \& Strathern, J. N. Yeast vital stains: visualizing vacuoles and endocytic compartments with FM4-64. Cold Spring Harb. Protoc. 2006, pdb.prot4165 (2006).

60. Wallace, W., Schaefer, L. H. \& Swedlow, J. R. A workingperson's guide to deconvolution in light microscopy. Biotechniques 31, 1076-1078, 1080, 1082 passim (2001).

61. Haas, B. J. et al. De novo transcript sequence reconstruction from RNA-seq using the Trinity platform for reference generation and analysis. Nat. Protoc. 8, 1494-1512 (2013).

62. Bryant, D. M. et al. A tissue-mapped axolotl de novo transcriptome enables identification of limb regeneration factors. Cell Rep. 18, 762-776 (2017).

63. Matasci, N. et al. Data access for the 1,000 Plants (1KP) project. Gigascience 3, 17 (2014). 


\section{Article}

64. Källberg, M. et al. Template-based protein structure modeling using the RaptorX web server. Nat. Protoc. 7, 1511-1522 (2012)

65. Bomati, E. K. \& Noel, J. P. Structural and kinetic basis for substrate selectivity in Populus tremuloides sinapyl alcohol dehydrogenase. Plant Cell 17, 1598-1611 (2005).

66. Weigel, D. \& Glazebrook, J. Transformation of agrobacterium using the freeze-thaw method. Cold Spring Harb. Protoc. 2006, pdb.prot4666-pdb.prot4666 (2006)

67. Kushnirov, V. V. Rapid and reliable protein extraction from yeast. Yeast 16, 857-860 (2000).

Acknowledgements We thank A. Cravens for the yeast multiplex CRISPR-Cas9/sgRNA plasmids (pCS3410, 3411, 3414 and 3700-3703); T. Lozanoski (Jarosz laboratory) for a plasmid containing yeast codon-optimized mVenus; J. Payne for assistance with the generation of DFT energy-optimized ligand structures for docking studies; P. Dykstra for assistance with fluorescence microscopy and the Stanford Cell Sciences Imaging Facility for access to microscopy equipment and training; R. Nett for suggestions regarding de novo transcriptome assembly using Trinity; W. Cody and the Sattely laboratory for providing N. benthamiana plants, Agrobacterium strains, a pEAQ-HT binary vector backbone plasmid, and suggestions for tobacco agroinfiltration experiments; D. Endy and J. Payne for discussions and feedback in the preparation of this manuscript. This work was supported by the National Institutes of Health and the Natural Sciences and Engineering Research Council of Canada (doctoral postgraduate scholarship to P.S.)

Author contributions P.S. and C.D.S. conceived of the project, designed the experiments, analysed the results, and wrote the manuscript. P.S. performed the experiments.

Competing interests P.S. and C.D.S. are inventors on a pending patent application. C.D.S. is a founder and CEO of Antheia, Inc.

Additional information

Supplementary information is available for this paper at https://doi.org/10.1038/s41586-020 2650-9.

Correspondence and requests for materials should be addressed to C.D.S.

Peer review information Nature thanks Jose Avalos, Oliver Kayser and the other anonymous reviewer(s) for their contribution to the peer review of this work. Peer reviewer reports are available.

Reprints and permissions information is available at http://www.nature.com/reprints. 


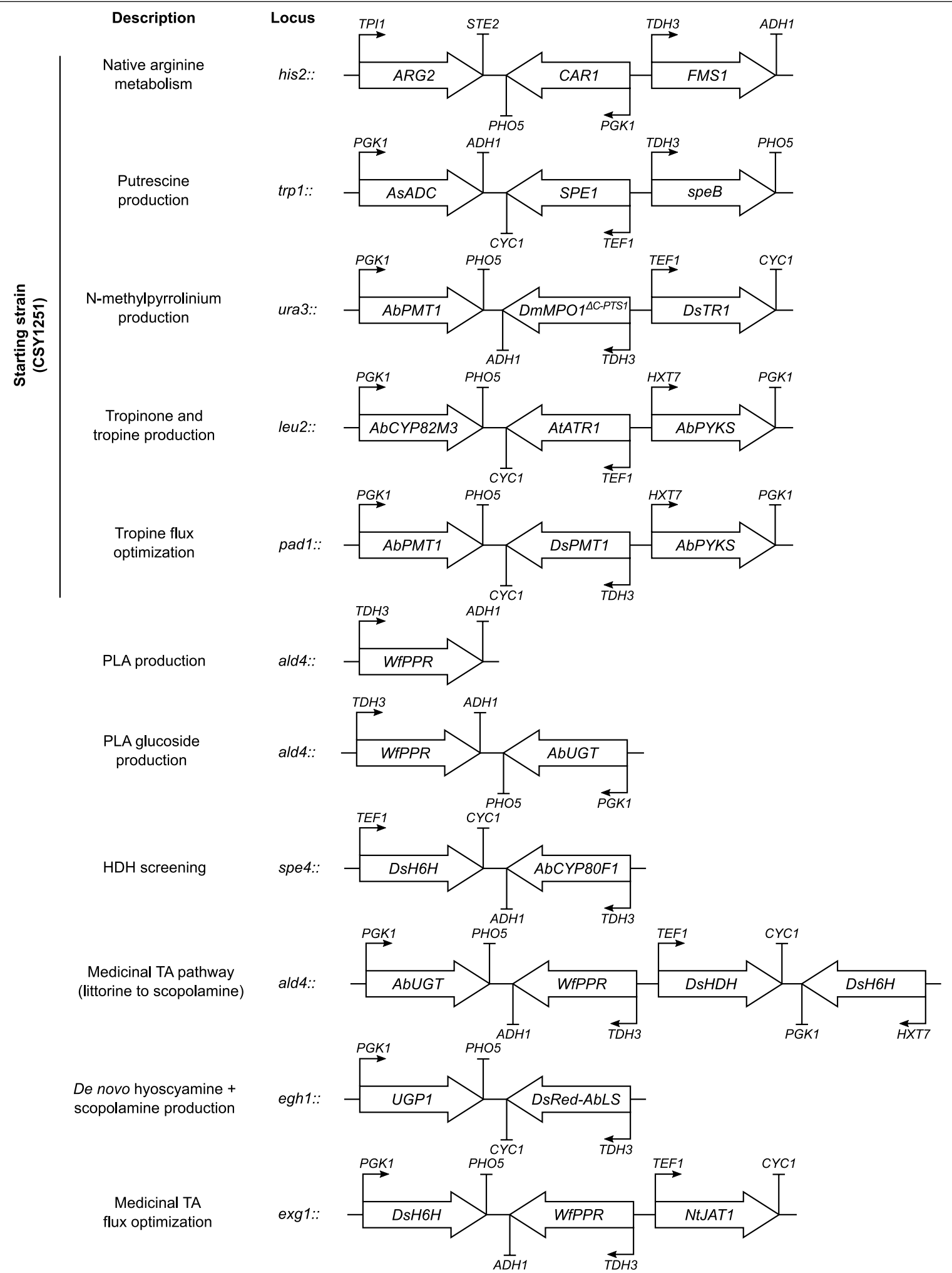

Extended Data Fig. 1 | Design of genomic integrations for pathway construction in yeast. Block arrows represent gene expression cassettes with unique promoter and terminator for each locus. Genus and species sources for heterologous genes are indicated by two letters preceding the gene symbol.
Superscript annotations on gene symbols indicate $\mathrm{N}$ - or C-terminal modifications; dash (-) indicates fusion protein. See Supplementary Tables $1-2$ for gene sources and Supplementary Table 3 for yeast strain genotypes. 


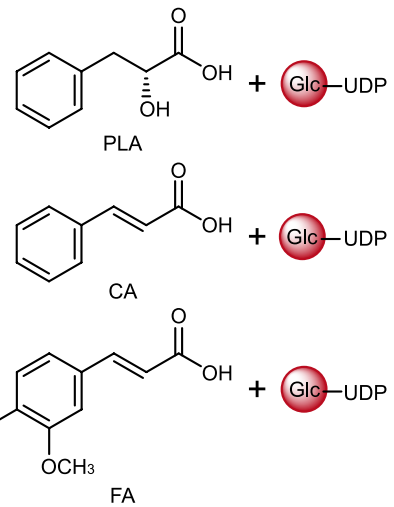

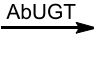<smiles></smiles><smiles>COC(=O)/C=C/c1ccc(O)c(OC)c1</smiles>

FA glucoside

b

\begin{tabular}{c|c|c|c|}
\multicolumn{1}{c}{} & \multicolumn{1}{c}{ PLA } & \multicolumn{1}{c}{ CA } & \multicolumn{1}{c}{ FA } \\
\cline { 2 - 4 } Control & 0.0 & 0.0 & 0.0 \\
& \pm & \pm & \pm \\
& 0.0 & 0.0 & 0.0 \\
\cline { 2 - 4 } AbUGT & 2.7 & 63.4 & 89.0 \\
& \pm & \pm & \pm \\
& 4.2 & 3.8 & 0.7 \\
\cline { 2 - 4 } BsUGT & 0.6 & 36.0 & 87.5 \\
& \pm & \pm & \pm \\
& 3.2 & 3.3 & 0.4 \\
\cline { 2 - 4 } DmUGT & 0.4 & 34.6 & 84.7 \\
& \pm & \pm & \pm \\
\cline { 2 - 4 } & 1.7 & 4.6 & 0.7 \\
\hline
\end{tabular}

e

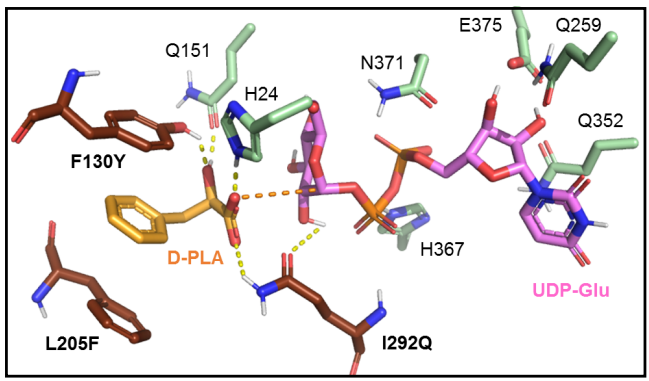

$\mathrm{CA}$

c

$E I C \mathrm{~m} / \mathrm{z}^{+} 184$
$\mathrm{EIC} \mathrm{m} / \mathrm{z}^{+} 346$

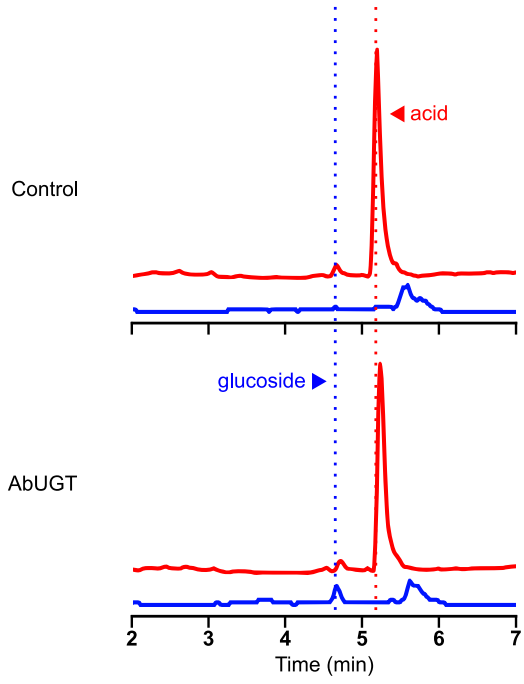<smiles>COC(=O)[C@H](O)Cc1ccccc1</smiles><smiles>COC(=O)/C=C/c1ccccc1</smiles>

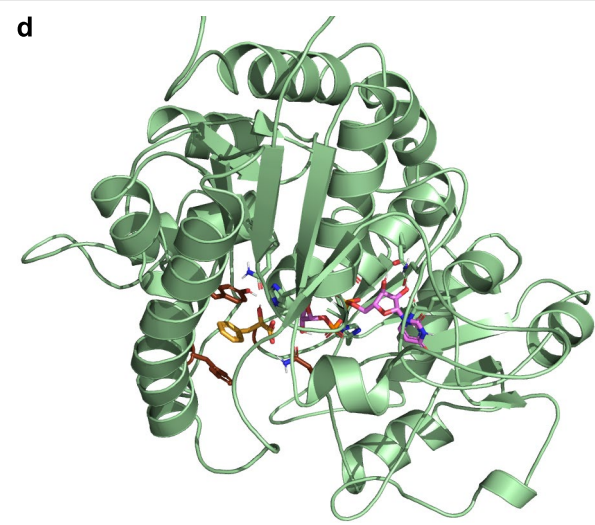

f

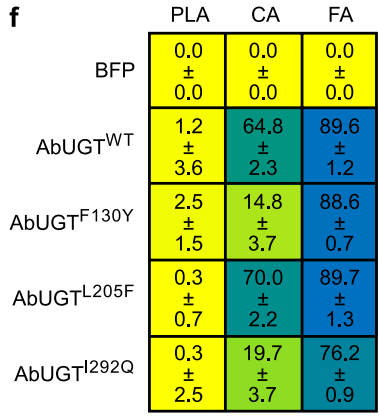

$\mathrm{FA}$ EIC $\mathrm{m} / \mathrm{z}^{+} 195$

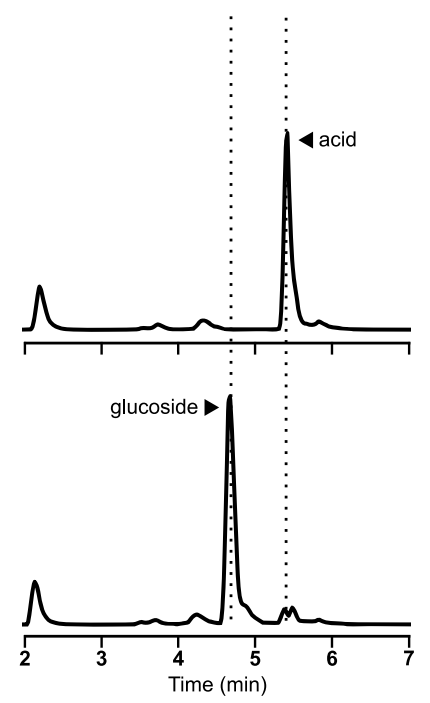

Extended Data Fig. 2 | Substrate specificity and structure-guided active site engineering of UGT 84A27 in engineered yeast. a, Phenylpropanoids tested as glucose (Glc) acceptors for UGT84A27 in engineered yeast. Top, (D)-3-phenyllactic acid (PLA); middle, trans-cinnamic acid (CA); bottom, trans-ferulic acid (FA).b, Heat map of the percentage conversion of fed phenylpropanoids to glucosides by yeast engineered for UGT84A27 expression. UGT84A27 orthologues or a BFP negative control were expressed from low-copy plasmids in CSY1251. Transformed cells were cultured in selective medium supplemented with $500 \mu \mathrm{M}$ PLA, CA or FA for $72 \mathrm{~h}$ before LC-MS/MS analysis of culture supernatant. Data represent the mean of $n=3$ biologically independent samples \pm s.d.c, Representative LC-MS/MS traces showing conversion of PLA, CA and FA to cognate glucosides by $A b U G T$ in CSY1251 cultured as in $\mathbf{b}$ for $120 \mathrm{~h}$ to enable more complete glucosylation. For PLA, acid and glucoside were distinguished by different $\mathrm{NH}_{4}{ }^{+}$adduct parent masses as well as different retention times. For CA and FA, rapid fragmentation necessitated detection of the glucosides based on the lower-retention peaks produced by their phenylpropanoid fragments. d, Homology model of AbUGT84A27 constructed based on the crystal structure of Arabidopsis thaliana salicylate UDP-glucosyltransferase UGT74F2 with bound UDP (PDB: $5 \mathrm{~V} 2 \mathrm{~K}$ ). PLA (orange) is shown in the preferred binding pose with UDP-glucose (pink) based on docking simulations. e, Zoomed view of $A b U G T$ active site with docked D-PLA and UDP-glucose. Potential mutations identified to improve PLA selectivity (F130Y, L205F, 1292Q) are shown; dashed lines indicate putative polar/hydrogen bond interactions. f, Heat map of the percentage conversion of fed phenylpropanoids to glucosides by yeast engineered for expression of $A b U G T$ mutants. $A b U G T$ wild-type, active site mutants, or a BFP negative control were expressed from low-copy plasmids in CSY1251. Transformed cells were cultured in selective media supplemented with $500 \mu \mathrm{M}$ PLA, CA or FA for $72 \mathrm{~h}$ before LC-MS/MS analysis of culture supernatant. Data represent the mean of $n=3$ biologically independent samples \pm s.d. 

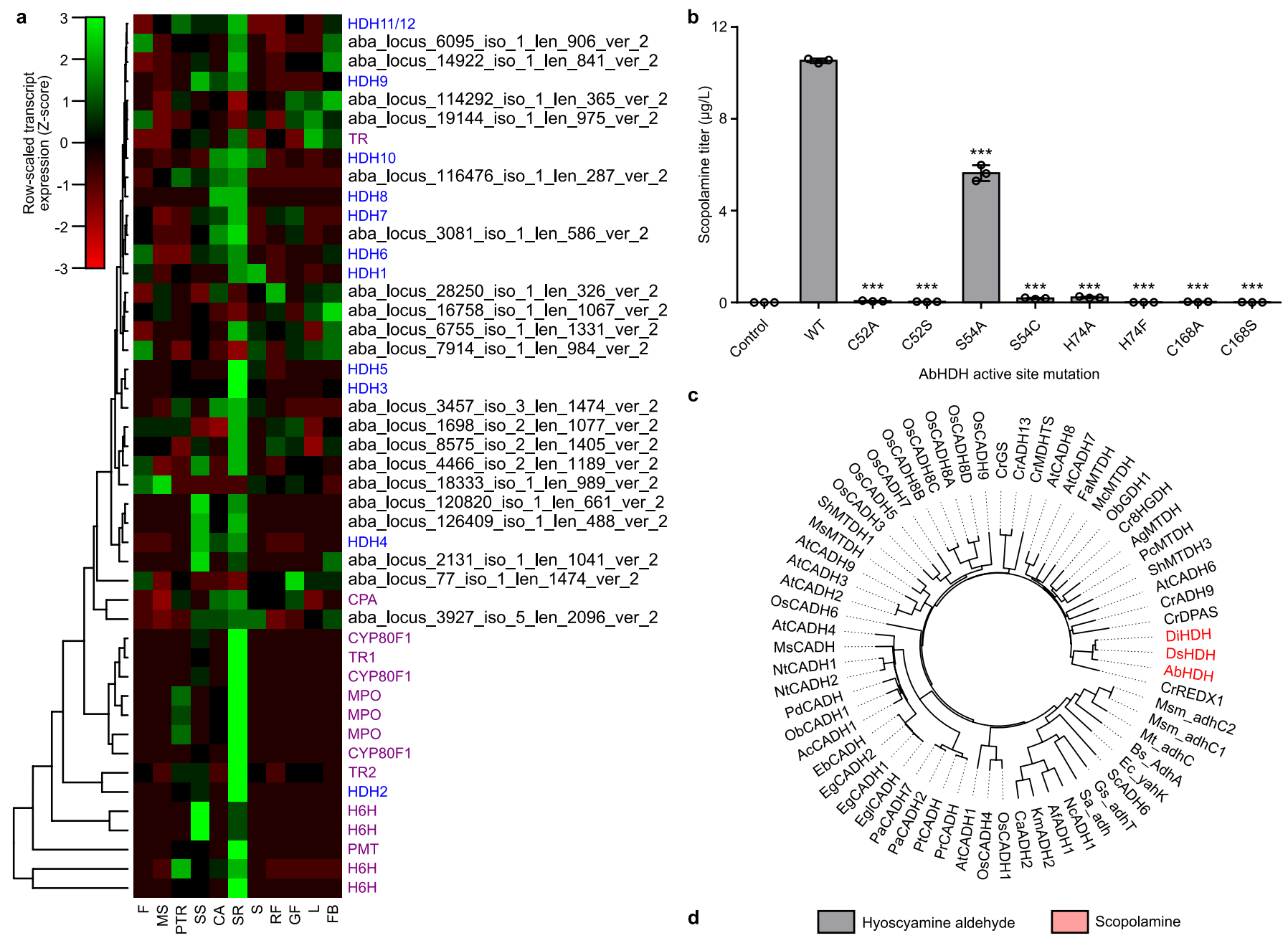

c

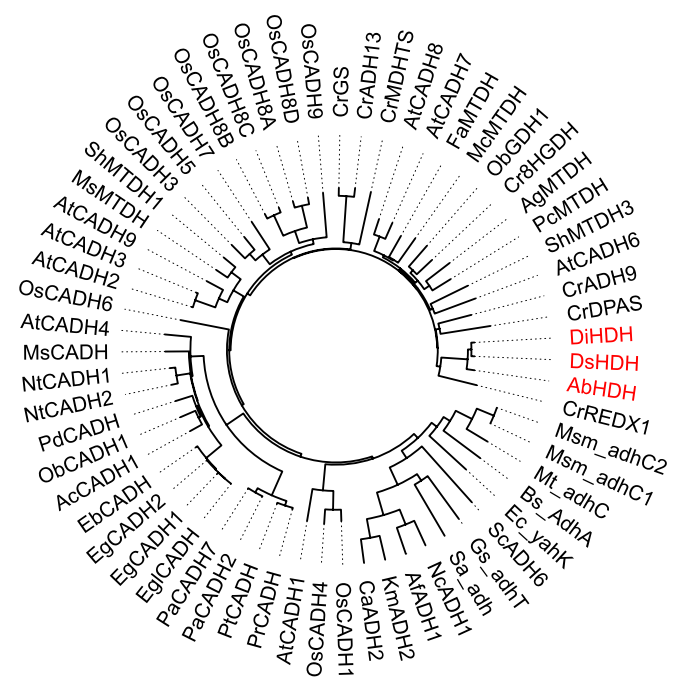

d

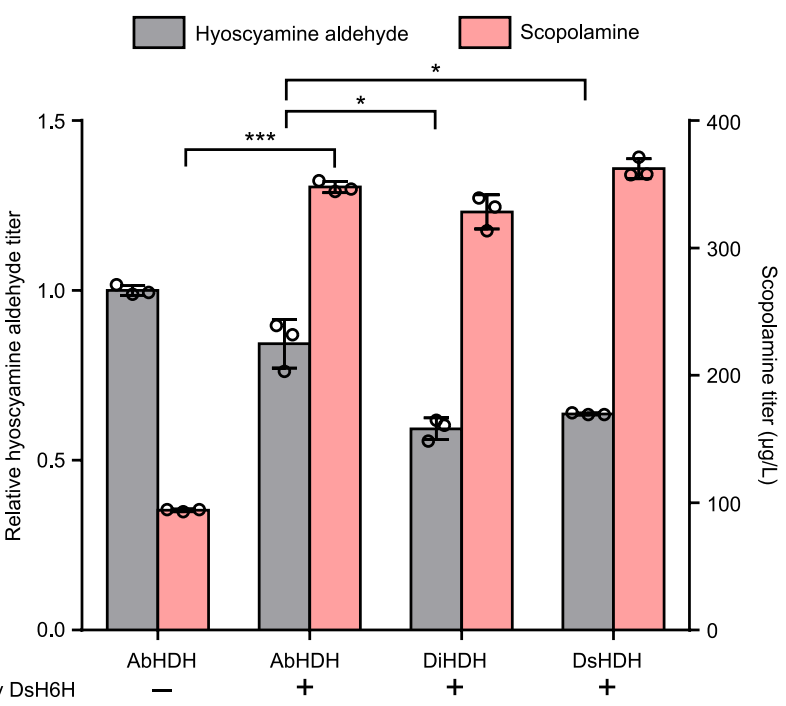

Extended Data Fig. 3 | See next page for caption. 


\section{Article}

Extended Data Fig. 3 | Coexpression analysis, active site mutagenesis, and orthologue identification for $\boldsymbol{A} \boldsymbol{b}$ HDH. a, Heat map of tissue-specific expression profiles for $\mathrm{HDH}$ gene candidates identified from the $A$. belladonna transcriptome. Transcript expression is scaled by row using a normal distribution. Dendrogram indicates hierarchical clustering of candidates by tissue-specific expression profile. Colour scheme for gene IDs: purple, known TA pathway genes; blue, putative HDH candidates with complete open reading frame sequences; black, putative $\mathrm{HDH}$ candidates with incomplete open reading frame sequences. Gene abbreviations (vertical axis): $\mathrm{CPA}, N$-carbamoylputrescine amidase. Tissue abbreviations (horizontal axis): F, flower; MS, mature seed; PTR, primary tap root; SS, sterile seedling; CA, callus; SR, secondary root; S, stem; RF, ripe fruit; GF, green fruit; L, leaf; FB, flower bud. b, Wild-type (WT) $A b \mathrm{HDH}$, active site mutants, or a negative control (BFP) were expressed from low-copy plasmids in CSY1292. Transformed strains were cultured in selective media with $1 \mathrm{mM}$ littorine at $25^{\circ} \mathrm{C}$ for $72 \mathrm{~h}$ before quantification of scopolamine production by LC-MS/MS analysis of culture supernatant. Data indicate the mean of $n=3$ biologically independent samples (open circles) and error bars show s.d. ${ }^{*} P<0.05,{ }^{* *} P<0.01,{ }^{* * *} P<0.001$, Student's two-tailed $t$-test. Statistical significance is shown relative to wild type. C52A, $P=1.68 \times 10^{-5}$; $52 \mathrm{~S}, P=2.06 \times 10^{-5}$; $54 \mathrm{~A}, P=8.19 \times 10^{-4}$; $54 \mathrm{C}, P=5.27 \times 10^{-6}$;
H74A, $P=3.40 \times 10^{-6} ; \mathrm{H} 74 \mathrm{~F}, P=2.78 \times 10^{-5} ; \mathrm{C} 168 \mathrm{~A}, P=1.75 \times 10^{-5} ; \mathrm{C} 168 \mathrm{~S}$ $P=2.39 \times 10^{-5}$.c, Phylogenetic tree of the three identified HDH orthologues $(A b \mathrm{HDH}, \mathrm{DiHDH}, \mathrm{DsHDH})$ together with closest protein hits in the UniProt/ SwissProt database. Phylogenetic relationships were derived via bootstrap neighbour-joining with $n=1,000$ trials in ClustalX 2 and the resulting tree was visualized with FigTree software. 8HGDH, 8-hydroxygeraniol dehydrogenase; $\mathrm{ADH}$, alcohol dehydrogenase; $\mathrm{CADH}$, cinnamyl alcohol dehydrogenase; DPAS, dehydroprecondylocarpine acetate synthase; GDH, geraniol dehydrogenase; GS, geissoschizine synthase; MTDH, mannitol dehydrogenase; REDX, unspecified redox protein. d, $\mathrm{HDH}$ orthologues $(A b \mathrm{HDH}, \mathrm{DiHDH}$ and $D s \mathrm{HDH})$ were coexpressed with either a BFP negative control ('-') or an additional copy of $D s \mathrm{H} 6 \mathrm{H}$ ('+') from low-copy plasmids in CSY1292. Transformed cells were cultured in selective medium supplemented with $1 \mathrm{mM}$ littorine for $72 \mathrm{~h}$ before $\mathrm{LC}-\mathrm{MS} / \mathrm{MS}$ analysis of culture supernatant. Data represent the mean of $n=3$ biologically independent samples (open circles) and error bars show s.d. ${ }^{*} P<0.05,{ }^{* *} P<0.01$, ${ }^{* * *} P<0.001$, Student's two-tailed $t$-test. $A b \mathrm{HDH}+D s \mathrm{H} 6 \mathrm{H}$ versus $A b \mathrm{HDH}$ only, scopolamine, $P=4.68 \times 10^{-5}$; $D i \mathrm{HDH}$ versus $A b \mathrm{HDH}$, hyoscyamine aldehyde, $P=0.0141 ; D s \mathrm{HDH}$ versus $A b \mathrm{HDH}$, hyoscyamine aldehyde, $P=0.0372$. 


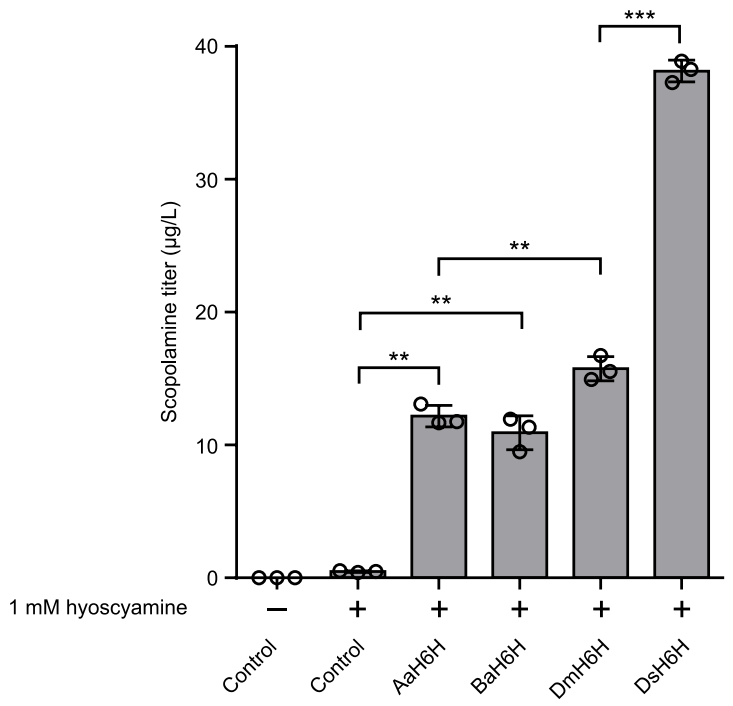

Extended Data Fig. 4 | Screening H6H orthologues from TA-producing Solanaceae in yeast. $\mathrm{H} 6 \mathrm{H}$ orthologues from Anisodus acutangulus $(A a \mathrm{H} 6 \mathrm{H})$, Brugmansia arborea $(B a \mathrm{H} 6 \mathrm{H})$, Datura metel $($ Dm $\mathrm{H} 6 \mathrm{H})$, Datura stramonium $(D s \mathrm{H} 6 \mathrm{H})$, or a negative control (BFP) were expressed from low-copy plasmids in CSY1251. Transformed cells were cultured in selective media supplemented with $1 \mathrm{mM}$ hyoscyamine for $72 \mathrm{~h}$ before LC-MS/MS analysis of culture supernatant. Data represent the mean of $n=3$ biologically independent samples (open circles) and error bars show s.d. ${ }^{*} P<0.05,{ }^{* *} P<0.01,{ }^{* * *} P<0.001$, Student's two-tailed $t$-test. $A a \mathrm{H} 6 \mathrm{H}$ versus control, $P=0.00146 ; B a \mathrm{H} 6 \mathrm{H}$ versus control, $P=0.00486 ; D m \mathrm{H} 6 \mathrm{H}$ versus $A a \mathrm{H} 6 \mathrm{H}, P=0.00739 ; D s \mathrm{H} 6 \mathrm{H}$ versus $D m \mathrm{H} 6 \mathrm{H}, 6.67 \times 10^{-6}$. 
a Ocimum_basilicum_CADH 1 Nicotiana tabacum CADH 1 Eucalyptus gunni ${ }^{2}$ CADH 2 Populus deltoides_CADH Arabidopsis_thaliana CADH4 Fragaria ananassa MTDH Medicago_sativa MTDH Catharanthus_roseus_8HGDH Ocimumbasilicum GDH 1 Catharanthus roseus GS AbHDH DiHDH

DSHDH

Ocimum_basilicum_CADH1 Nicotiana_tabacum_CADH 1 Eucalyptus_gunnii_CADH2 Populus_deltoides_CADH Arabidopsis_thaliana_CADH Fragaria_ananassa_MTD Medicago_sativa_MTDH Catharanthus_roseus_8HGDH Ocimum_basilicum_GDH1 Catharānthus_roséus_GS AbHDH

DiHDH

DSHDH

Ocimum_basilicum_CADH 1 Nicotiana_tabacum_CADH 1 Eucalyptus_gunnii_CADH2 PrabicoFragaria_ananassa_MTDH Medicago sativa MTDH Medicago sativa MTDH
Ocimumbasilicum_GDH1 Catharanthus_roseus_GS

AbHDH

DiHDH

Ocimum_basilicum_CADH1 Nicotiāna_tabacum_CADH 1 Eucalyptus_gunnii_CADH2 Populus_deltoides_CADH Arabidopsis_thaliana_CADH Fragaria_ananassa_MTDH Medicago_sativa_MTTH Catharanthus_roseus_8HGDH Ocimum_basilicum_GDH1 Catharanthus_roseus_GS AbHDH DiHDH DSHDH

Ocimum_basilicum_CADH1 Nicotiāna tabacum CADH 1 Eucalyptus gunnii_CADH2 Populus del toides CADH Arabidopsis thaliana_CADH Fragaria_ananassa_MTD Medicago_sativa MT̄DH Catharanthus_roseus_8HGDH CCImum_basilicum_GDH 1 Cathar AbHDH DiHDH
DSHDH
-MG-SLEV--ERKTVGWAARDP S GVLS PYEYTLRNTG PQDVYVEVMCCG ICHTDVHQ I KNDLGMSNY PMVPGHEVVGEV MG-GLEV--EKTTIIGWAARDPSGVLSPYTYTLRNTGPEDVEVKVLYCGLCHTDLHOVKNDLGMSNYPLVPGHEVVGEV -MG - S LEK--ERTT T GWAARD P S GV LS P Y T Y S LRNT G PE D LY I KV L SC GVCHS D I HQ I KN D L GMS HY PMV P GHEVVGEV

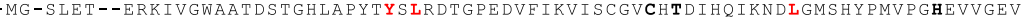

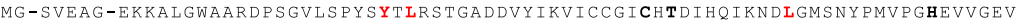
-MAIEQEHRKKAS GWAARDSS GVLS PFN FYRRET TEK DVT FKV LYCG ICHS DLHMVKNEW G S T Y P LVP GHE IVGEV MAKS PETELPLKAFGWAARDT SGT LSPFHFSRRENG D D DVSVK I LYCGVCHS DLHTLKN DWGFTTY PVVPGHEIVGVV - MAKS PEVEHPVKAFGWAARDT SGHLSPFHFSRRATGEHDVQFKVLYCG ICHS DLHMIKNEWGFTKYP IVPGHEIVG IV - -MAKSPETEHPVKALGWAAT DNS GTFS PFNFSRRATGER DVQFRV LYCGVCHS DLHMVKNEWGVTHYPIVPGHEIVGIV -MAGETTKLDLSVKAVGWGAADA S GVLQP I KFYRRVP GERDVK I RVLY S GVCNF DMEMVRNKWGFTRYPYVFGHETAGEV MASEKS LEEKQAENTFGWAAMDS S GVLS PFTFSRRATGEEDVRLKVLYCG ICHSDLGC I KNEWGWCSYPLVPGHEIVG IA MAAEK - LSEEEAVKT TGWAAMDS S GVLS PFEFSRRATGAEDVRLKVLYCG ICHS DLGCVKNEWGWCSYPLVPGHEIVGIA MAAEK-LEERKRWETEGWAAMDSS GVLSPFEFSRRATGEEDVRLKVLYCGICHSDLGC IKNEWGWCSYPLVPGHEIVGIA

VEVGSEVTKFRAGDVVGVGCIVGSCGNCRPCNSDIEQYCNKKIWSYN-DVYP-DGKPTQGGFAGAMVVDQKFVVKIPDGM VEVGPDVSKFKVGDTVGVGILVGSCRNCGPCKRDIEQYCNKK I WNCN - DVYT - DGKPTOGGFAKSMVVDQKFVVKI PEGM VEVGPDVSKFKVGDTVGVGLLVGSCRNCGPCKRDIEQYCNKKI WNCN-DVYT-DGKPTQGGFAKSMVVDQKFVVKIPEGM VEVGSDVTREKVG DVVGVGVIVGSCKNCHPCKSEIEOYCNKKIWSYN-DVYT - DGKPTOGGEAESMVVHOKEVVRIPDGM DV TEVGSKVQKFKVGDRVGVGCIVGSCRSCENCTDHLENYCPKQILTYG-AKYY-DGSTTYGGYSDIMVADTHFIVRIPDNL TKVGINVKKFRVGDNVGVGVIVESCOTCENCNODLEOYCPKPVFTYNS-PYK--GTRTYGGYSDFVVVHORYVVQFPDNL TEVGSKVEKFKVGDKVGVGCLVGSCRKCDMCTKDLENYCPGQILTYS-ATYT - DGTTTYGGYSDLMVADEHFVIRWPENL TEVGSKVEKVKIGDKVGVGVLVGSCRQCDQCSNDLENYCYKQILTYG-APYL-DGTIARGGYSDIMVADEHFIIRWPENF VEVGSKVEKFKVGDKVAVGCMVGSCGQCYNCQSGMENYCPEPNMADG-SVYREQGERSYGGCSNVMVVDEKFVLRPENL TEVGSKVTKFKVGDRVGVGCMVGSCGICQNCTQNQESYCP VIMTCA-SAYP-DGTPTYGGYSNQMVANEKFVIRIPNSL TEVGSRVTKFKVGDRVGVGCMVGSCGSCQNCSQNLESYCPEVIMTCA-SAYP-DGTPTYGGF SNQMVANEKFVIQI PEKL APEQAAPLLCAGVTVYSPLNHFGLKQ-SGLRGGILGLGGVGHMGVKIAKAMGHHVTVISSSDKKRAEALDHLGADDYLVS APE QAAP L LCAG I TVYS P LNHFGFKQ-SGLRGG I L GLGGVGHMGVK IAKAMGHHVTVISSSNKKRQEA LEH LGADDYLVS ESEQAAPLMCA GVTVY S PLVRFGLKQ-SGLRGGILGLGGVGHMGVK I AKAMGHHVTVISSSDKKRTEALEHLGADAYLVS SPEQAAPLICAGLTVYSPLKHFGLKQ-SGLRG G I G L G GV GHMGVK I AKAMGHHVTV I S S DKKREEAMEH LGADEYLVS AVEOAARLICAGVTVYS PLDGAAPLICAGITTYSPLRYFGLD-KPGMHVGVVGLGGLGHVAVKFAKAMGVKVTVISTS PKKEEEALKHLGADSFLVS PLDAGAPIICAGITVY SPMKYYGMT - EPGKHIGVAGIGGI GHVAIKFGKAFGLKVTVTSTS PNKETEATDKLGADSFIVS PMDIGAPLICAGITTYSPIRYFGID-KPGTHVGVVGLGGIGHVAVKFAKAFGAKVTVISTSESKKOEALEKLGADSFIVS PLDAGAPLLCAGITTYSPIKY FGID-KPGLRVGVNGLGGLGHVAVKFAKAFGTKVTVISTSISKKE EAMOHIGVDEFVVS PODKGVALICAGVVVY S PMKHI GID-KPGKH I GVFGLGGIGSVAVKF I KAFGGKATVIST SRRKEKE I T THGADAFVVN PIDAAAPLICAGSTVYSAMK FYGLC-SOGLHLGVVGLGG GHVAVKFAKAFGMKVTVISTS IGKKE PIDAAAPLICAGSTVYSPMKEYGLC-SPGIHLGVVGLGGLGHVAVKFAKAFGMKVTVISTSIGKKEEAINOIGADSEITS

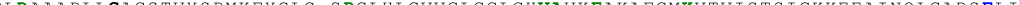

SDAARMQEAADSLDY I I DTVP-VFHPLEPYLSLLKID-GKL I LMGVVNT PLQFVS PMVMLGRKS ITGSFIGSMKELAEML S D T DKMQEAS DS L DY I I D TVP-VGH P LE PY L S L LK I D-GKL I LMGV IN T P L QF I S PMVMLGRKS I T GSF I GSMKE TEEML SDENGMKEAT DS LDY I F DT I P-VVHPLE PY LALLKLD-GKL I LTGVINA P LQF I S PMVMLGRKS ITGSFIGSMKETEEML SDVESMQKAADQLDYIIDTVP-VVHPLEPYLSLLKLD-GKLILMGVINAPLQFVT PMVMLGRKSITGSFIGSMKETEEML SDPAEMQRLADSLDYIIDTVP-VRHPLDPYLACLKLD-GKLILMGVINIPLQFVIPLVILGRKVISGSFIGSIKEIEEVL RDQDQMQAAIGTMDGIIDIVS-AQHPLLPLIGLLNSH-GKLVMVGAPEKPLELPVFPLIMGRKMVAGSGIGGMKETQEMI KDPEKMKAAMGTMDYIIDTIS-AAHSLMPLLGLLKLN-GKLVTVGLPSKPLELSVFPLVAGRKLIGGSNIGGMKETQEMI RDPEQMKAAAASLDGIIDTVS-AIHPIMPLLSILKSH-GKLILVGAPEKPLELPSFPLIAGRKIIAGSAIGGLKETQEMI TDPQQMQAAVGTLDGIIDTVS-APHPIVPLLSLLKPH-GKLIVVGLPDKPLQLPVFPLIQGRRTIAGSGIGGLKETQEMI TDSEQLKALAGTMDGVVDTTPGGRT PMS LMLNLLKFD-GAVMLVGAPESLFELPAAP LIMGRKKII GSSTGGLKEYQEML TDTEQMQGAMEVMDG I IDTVS - ALHP IEPLLGLLKSHQGKLI IVG LPNKQPE LPVFS I NGRKMI GGSAVGGVKE TQEM I TDTEQMQGAMETMDG I IDTVS - A LHP IEPLVGLLKSHQGKLI IVG LPNKQPE LPVES L INGRKMI GGSAVGGVKETQEMI TDTEQMQGAME TMDG I I DTVS - ALH P I P LVGLLKSHRGKL I IVGL PNKQPE L PVES L INGRKMI GGSAVGGVKETQEMI EFCKEKDLSSTIEIVKMDYINTAFERLEKNDVRYRFVVDVAGSKLYQ------ 357 DFCKEKGVTSQIEIVKMDYINTAMERLEKNDVRYRFVVDVIGSKLDQ----- 357

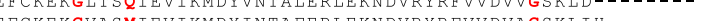
AFCKEKGITSTIETVRIDEINIAFERIRKNDVRYREVVDVRGSNIVEEAATTTN DFAARHN I TAD IEV I PI DY LNT AMER LVKADVRYRFVIDIGNT LKVRS - - - 35 DFCGKHNITADIEL I KMHEINTAMERLHKADVKYRFVIDVANSESSL-- - - - 359 DFAAKHNVL PDVELVSMDYVNTAMERLLKADVKYRFVIDVANTLKSA----- $\quad 360$

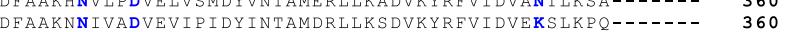
DFAAKHNIVCDTEVIGIDYLSTAMERIKNLDVKYRFAIDIGNTLKFEE---- 364 DFAAE HNI TAD IE I VPMDYVNTAMERLEKGDVKFRFVIDVENTLVAAQT---- $\quad 365$ $\begin{array}{ll}\text { DFAAKHNITADI I I VRMDYVNT AMERLEKG DVKFRFVIDVENTLVPAQT----- } & \mathbf{3 6 4}\end{array}$ DFAAKHNITADIE IVGMDYVNTAMERLEKGDVKFRFVIDVENTLVPAQT-_.-- - 364
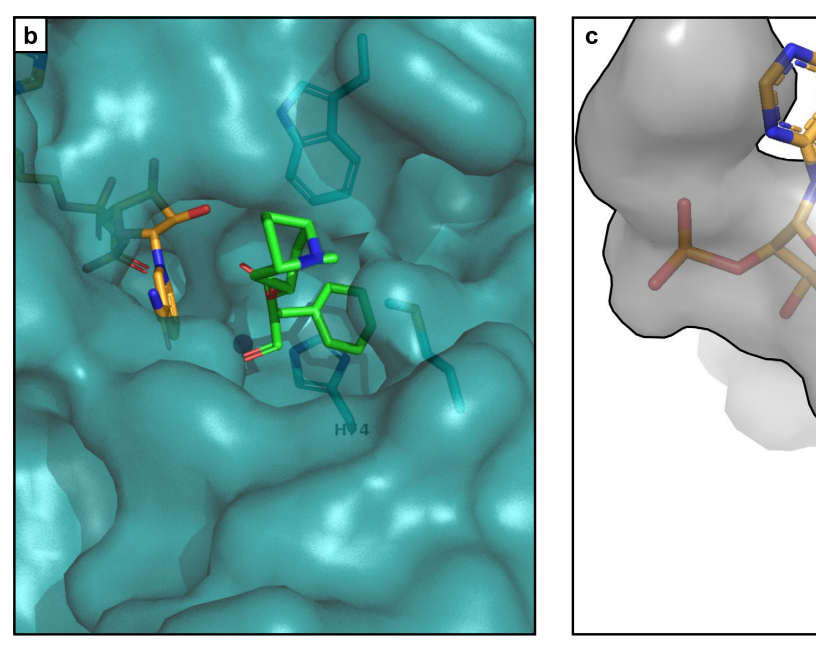

Extended Data Fig. 5 | Phylogenetic analysis of HDH. a, Sequence alignment of $A b \mathrm{HDH}, D i \mathrm{HDH}$ and $D s \mathrm{HDH}$ (green) with selected BLASTp hits from the cinnamyl alcohol dehydrogenase (CAD)-like (red) and sinapyl alcohol dehydrogenase (SAD)-like (blue) families. Catalytic $\mathrm{Zn}^{2+}$ residues are highlighted in bold (black); structural $\mathrm{Zn}^{2+}$-binding residues are highlighted in pink; and key conserved residues which differentiate $C A D$ - and SAD-like $A D H s$ are highlighted in red/blue/ green. $\mathbf{b}$, Zoomed surface view of $A b H D H$ substrate-binding pocket with bound

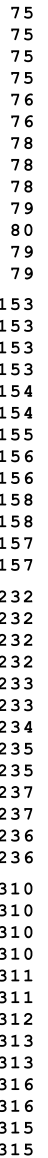




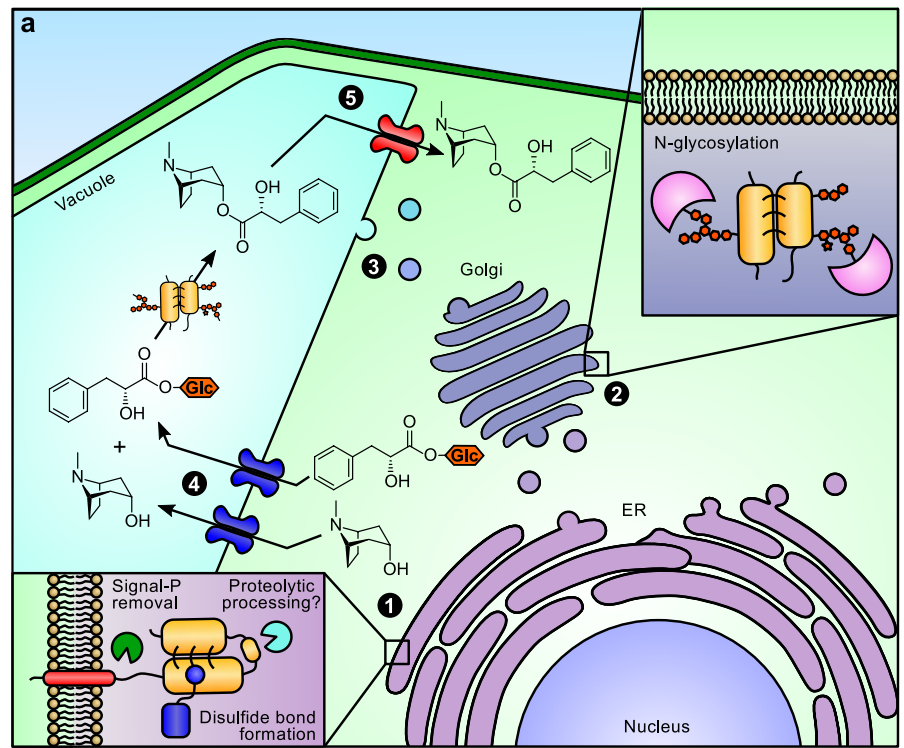

c

$$
\text { d }
$$

Host:

N152Q $\frac{\text { Yeast }}{-++}+-\frac{N b}{-}+-$

$\mathrm{N} 320 \mathrm{Q}-++\quad+-+-$

Deglycosylation: - $\quad N \quad 0$

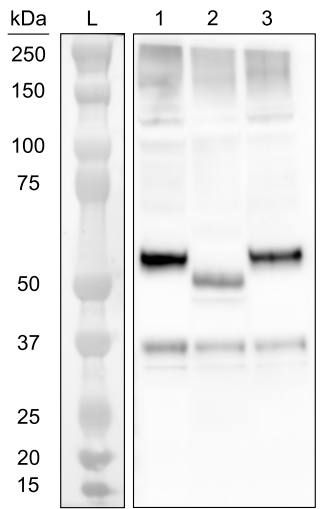

f

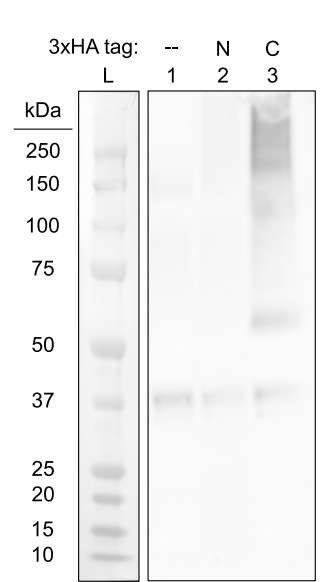

g

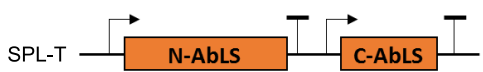

\section{b}

GFP-AbLS

FM4-64

(vacuoles)
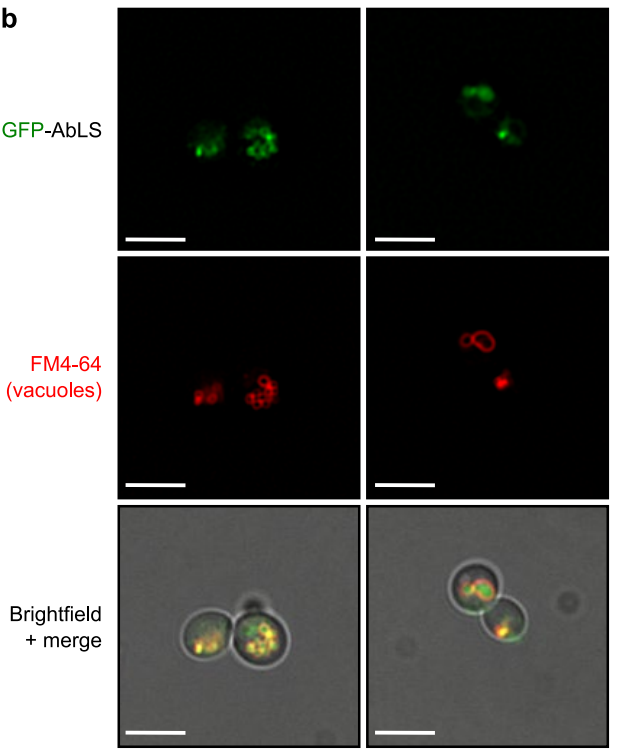

e
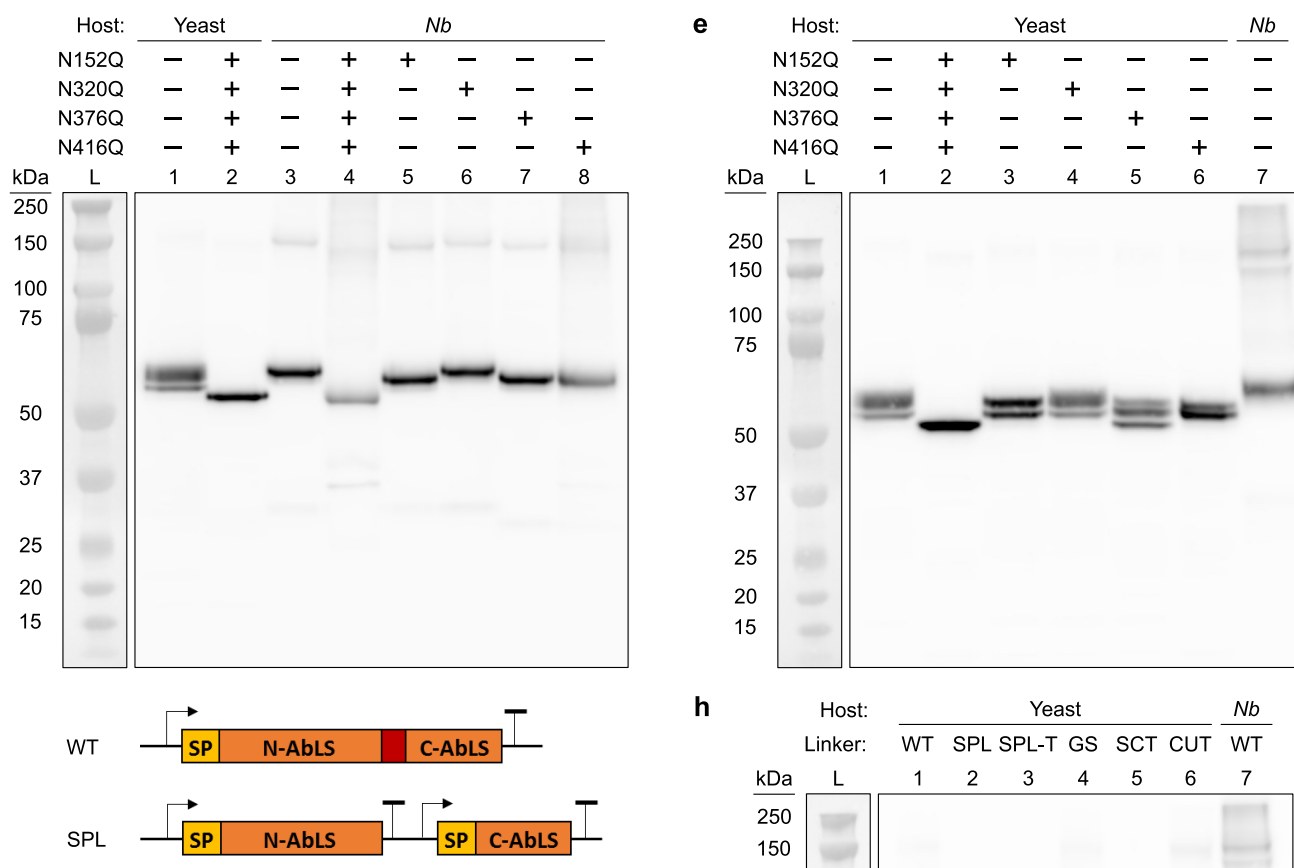

GS \begin{tabular}{r|c|c|c|}
\hline SP & N-AbLS & (GGGGS) & C-AbLS \\
\hline
\end{tabular}

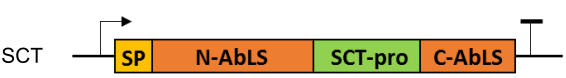

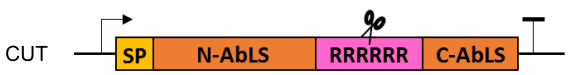

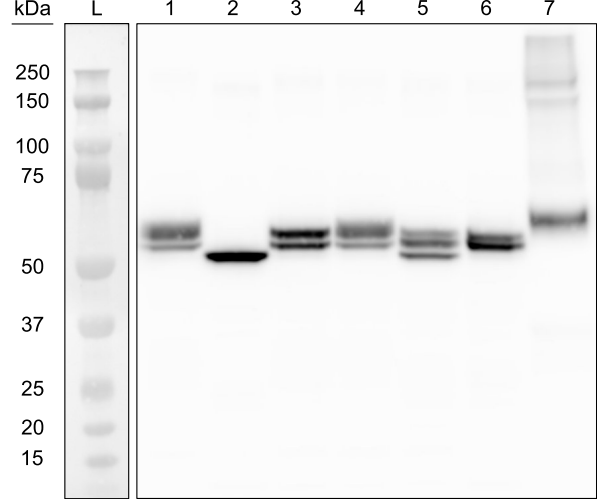

h

\begin{tabular}{rlr} 
Host: & \multicolumn{1}{c}{ Yeast } & $N b$ \\
Linker: & WT SPL SPL-T GS SCT CUT & WT
\end{tabular}

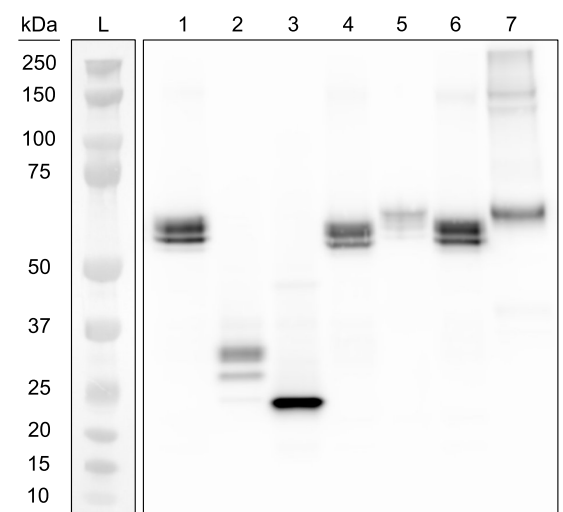

Extended Data Fig. 6 | See next page for caption. 


\section{Article}

Extended Data Fig. 6 | Analysis of $\boldsymbol{A} \boldsymbol{b}$ LS localization, $\mathrm{N}$-glycosylation, and proteolytic processing patterns in yeast and tobacco. a, Illustration of the canonical plant ER-to-vacuole trafficking and maturation pathway for SCPL acyltransferases (SCPL-ATs), with $A$. belladonna littorine synthase ( $A b \mathrm{LS}$ ) shown as example. Circled numbers indicate major steps in SCPL-AT expression and activity, including maturation in the (1) ER lumen and (2) Golgi, (3) trafficking to the vacuole, and vacuolar (4) substrate import and (5) product export.

b, Additional fields of view (see Fig. 3a) of yeast epifluorescence microscopy showing $\mathrm{N}$-terminal GFP-tagged $A b \mathrm{LS}$ (GFP- $A b \mathrm{LS}$ ), the vacuolar membrane stain FM4-64, and brightfield merged images. Microscopy was performed on CSY1294 expressing GFP- $A b$ LS from a low-copy plasmid. 2D deconvolution analysis was performed as noted in the Methods. Scale bar, $5 \mu \mathrm{m}$. Images are representative of two independent experiments. c, Western blot of wild-type $A b$ LS expressed in tobacco and treated with deglycosylases. C-terminal HA-tagged $A b$ LS was transiently expressed in $N$. benthamiana leaves via agroinfiltration. Crude leaf extracts were either untreated (lane $1:^{\prime}{ }^{\prime}$ '), or treated with peptide $N$-glycosidase $F$ (PNGase F; lane 2: ' $\mathrm{N}$ ') or $O$-glycosidase (lane 3: ' $\mathrm{O}$ ') to remove $\mathrm{N}$ - or $\mathrm{O}$-linked glycosylation, respectively. Lane 'L', Bio-Rad Precision Plus Dual Colour protein ladder. d, e, Western blot of $A b$ LS glycosylation site mutants expressed in yeast and tobacco. C-terminal HA-tagged wild-type $A b \mathrm{LS}$, single glycosylation site point mutants ( $\mathrm{N}$ to $\mathrm{Q}$ ), or a quadruple mutant were expressed transiently via agroinfiltration in $N$. benthamiana ('Nb') (d) or from low-copy plasmids in CSY1294 ('yeast') (e). For d and e, corresponding yeast- and tobacco-expressed controls are included for comparison. Lane ' $\mathrm{L}$ ', Bio-Rad Precision Plus Dual Colour protein ladder.f, Western blot of untagged and HA-tagged wild-type $A b \mathrm{LS}$ expressed in tobacco. Untagged (lane 1:'-'), N-terminal HA-tagged (lane 2: 'N'), or C-terminal HA-tagged (lane 3: ' $C$ ') $A b$ LS was transiently expressed in $N$. benthamiana leaves via agroinfiltration. Lane ' $\mathrm{L}$ ', Bio-Rad Precision Plus Dual Colour protein ladder. $\mathbf{g}, \mathbf{h}$, Analysis of proteolytic cleavage patterns for $A b \mathrm{LS}$ split controls and putative propeptide-swapped variants $(\mathbf{g})$ in yeast via western blot (h). C-terminal HA-tagged $A b \mathrm{LS}$ variants were expressed from low-copy plasmids in CSY1294 (lanes 1-6); HA-tagged wild-type $A b$ LS expressed in Nicotiana benthamiana $(\mathrm{Nb})$ is shown as an additional control (lane 7). Lane symbols: $\mathrm{L}$, protein molecular mass ladder; WT, wild-type $A b \mathrm{LS}$; SPL, $A b \mathrm{LS}$ split at putative propeptide with signal peptides on both fragments; SPL-T, $A b \mathrm{LS}$ split at putative propeptide without signal peptides on either fragment; GS, $A b$ LS variant with wild-type propeptide swapped for flexible Gly-Ser linker; SCT, $A b$ LS variant with wild-type propeptide swapped for $A t S C T$ propeptide sequence; CUT, $A b$ LS variant with wild-type propeptide swapped for synthetic poly-arginine site recognized and cleaved by Kex2p protease. For blots in $\mathbf{c}-\mathbf{h}$, sample preparation, electrophoresis and protein transfer steps were performed under denaturing and disulfide-reducing (c-e, h) or non-reducing (f) conditions; $A b$ LS detection was performed using a chimeric rabbit IgGK anti-HA HRP-conjugated antibody (Methods). Blots are representative of three $(\mathbf{c})$ or two $(\mathbf{d}-\mathbf{f}, \mathbf{h})$ independent experiments. For gel source data, see Supplementary Fig. 1. 


$\begin{array}{ll}\text { a } & \text { AtSCT } \\ \text { AtSMT } \\ \text { AbLS } \\ \text { AsSCPL1 } \\ \text { TaCBP2 } \\ \text { yPRC1 } \\ \\ \text { AtSCT } \\ \text { AtSMT } \\ \text { AbLS } \\ \text { AsSCPL1 } \\ \text { TaCBP2 } \\ \text { yPRC1 } \\ \\ \text { AtSCT } \\ \text { AtSMT } \\ \text { AbLS } \\ \text { AsSCPL1 } \\ \text { TaCBP2 } \\ \text { yPRC1 } \\ \\ \text { AtSCT } \\ \text { AtSMT } \\ \text { AbLS } \\ \text { AsSCPL1 } \\ \text { TaCBP2 } \\ \text { yPRC1 } \\ \text { AtSCT } \\ \text { AtSMT } \\ \text { AbLS } \\ \text { AsSCPL1 } \\ \text { TaCBP2 } \\ \text { yPRC1 } \\ \text { AtSCT } \\ \text { AtSMT } \\ \text { AbLS } \\ \text { AsSCPL1 } \\ \text { TaCBP2 } \\ \text { yPRC1 } \\ \text { AtSCT } \\ \text { AtSMT } \\ \text { AbLS } \\ \text { AsSCPL1 } \\ \text { TaCBP2 } \\ \text { yPRC1 } \\ \text { AtSCT } \\ \text { AtSMT } \\ \text { AbLS } \\ \text { AsSCPL1 } \\ \text { TaCBP2 } \\ \text { yPRC1 } \\ \text { Y } \\ \end{array}$

b

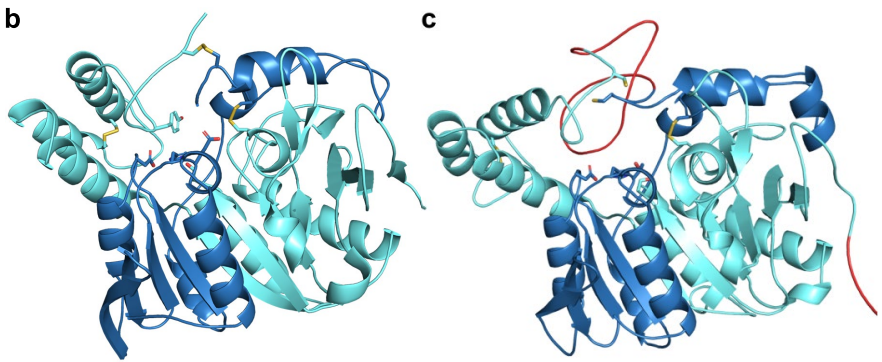

Extended Data Fig. 7 | Analysis of putative endoproteolytic propeptide removal in $\boldsymbol{A} \boldsymbol{b} \mathbf{L} S$. a, Sequence alignment of $A b \mathrm{LS}$ with characterized serine carboxypeptidases and SCPL acyltransferases known to possess (AtSCT, AsSCPL1, TaCBP2) or lack (AtSMT, yPRC1) proteolytically-removed internal propeptide linkers (red). Putative $\mathrm{N}$-terminal signal peptides are indicated in bold; disulfide bonds are indicated in blue. AtSCT, Arabidopsis thaliana sinapoylglucose:choline sinapoyltransferase; AtSMT, A. thaliana sinapoylglucose:malate sinapoyltransferase; $A b \mathrm{LS}$, Atropa belladonna littorine synthase; AsSCPL1, Avena strigosa avenacin synthase; TaCBP2, Triticum

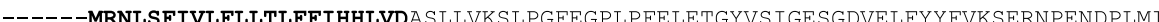
MEKLLVVVLLLVTILALGAAAERTRVTHLKGFDGPLPFSLETGYVEVDETHGVELFYYFIESERKPAEDPVII 作 WVSGGPGCSGLN-ALFFEIGPLKLDMASYAATGGKGFP GLLYFEDAWTKASNMIFLDAPVGAGFSYARQTEGLNST-VTG WLNGGPGCS SVAYGASEELGAFRVKPR--------GAGLVLNEYRWNKVANVLFLDSPAGVGFSYTNTSSDIYTSGDNR EVKRTHEFLOKWLSRHPOYFSN--PLYVVGDSYSGMIVPALVQEISOGNYICCEPPINLQGYMLGNPVTYMDFEONFRIP MALHTHQFVQKWLVDHSEYLSN--DFYVAGDSYSGISVPI ITQVISDGNEAGNKPWINLKGYILGNAVTFRPDEQNYRIP LGRHVRVFLQKWMAQHPELASN--PLYIGGDSFSGYTVTVSALEVA--NHPAAS SELNLKGYMVGNARGEVNNDNACRIP TAHDSYAFLAKWFERFPHYKYR--DFYIAGESYAGHYVPELSOLVHR----SKNPVINLKGFMVGNGLIDDYHDYVGTFE

SDEFESLERSCGG---KFFNVDPSNARCSNNLQAYDHCMSEIYSEHILLRNC---YAYGMGLISDEIYEPMKRICNG---NYYNVDPSNTQCLKLTEEYHKCTAKINIHHILTPDC-YLHGMGLISDELYEAALSSCVVGTDSKNKQQQSAARCSEAQQA I SEATTDLNPAH I LEPACGADFSPRAPYLSLTTPSSS

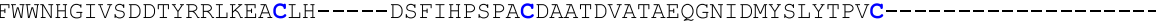
MACGEGGEPSVLPSEECSAMED---SLERCLGLIESCYDSQSVWSCVPAT IYCNNAQLAPY--------------

KVDYVLADTPNIRTDRRRVMKEFSVNDSSSLPPPSCFTYRYFLSAFWANDENVRRALGVKK-EVGK--WNRCNS-QNIPY DVTNVTS------NPVSTKARHLSPQR-RYLNQKLGQLKNPTMLPGVKCRDEWHLLSE IWVNDETVQEALHVRKGTHGI - -WKQCPNYEKMPF SSSSSSSSSSSSSYYYLSLSSVRSRTPTKEMLLECRVYGYELSYMWANDAEVRENLGVREGTIGDGNWALCPEVPKLHL NITSSSSSSSSSLS-----OORRSRGRYPWLTGSYDPCTERYSTAYYNRRDVQMALHANVTGAMNYTWATCSDTINTHW QRTGRNVYDIR----------------KDCEGGNLCYPTLQDIDDYLNQDYVKEAVGAEVDHYESCNFDINRNF

TFEI FNAVPYHVNNS---LKGFRSLIYSGDHDSMVPFSSTQAWIRALNYS IVDDWR-----PWMMSS-NQVAGYTRTYAN NHDIVSS I PYHMNNS---ISGYRS L IYSGDHDIAVPFLATQAWIRS LNYSP I HNWR---- -PWMIN --NQIAGYTRAYSN TRT INNT I PFHAS LS -- -KKGYRS L IYS GDYDLYVPFLSTOAWIRS LNYS I DTEWR-- - - RWFVD - -GQVAGYVTTYSN TNDVPTTVPYHRRLT---QRGYRALVYNGDHDLLMTH I GTHAW IRSLGYPVVAPWR-----AWYSN--NEVAGFTVEYSN HDAPRSMLPIYRELI---AAGLRIWVFSGDTDAVVPLTATRYS IGALGLPTTTSWY-----PWYDD--QEVGGWSQVYKG LFAGDWMKPYHTAVTDLLNQDLP I LVYAGDKDF I CNWLGNKAWTDVLPWKYDEEFASQKVRNWTAS ITDEVAGEVKSYKH
80

74

70

84
73

57

159

148

143

158

151
128

225

226

221

236

227

202

284

279

294

307

359

360

329

371

371

387

332
417

431

431

399

441

457
402

497
KMTFATIKGGGHTA-EYTPDOCSLMFRRWIDGEPL_--_--KMTFATIKGGGHTA-EYRPNETFIMFQRWISGQPL-------- 433 QMTETTIKGAGHTAPEYKPAECLAMLKRWIYYQPL-------- 476 NLTFATVKGAGHMAPESRPKQCLDMVRRWISPAGKL------- 493 -LTLVSVRGAGHEVPLHRPRQALVLFQYFLQGKPMPGQTKNAT 444 -FTYLRVFNGGHMVPFDVPENALSMVNEWIHGGFSL------- 532
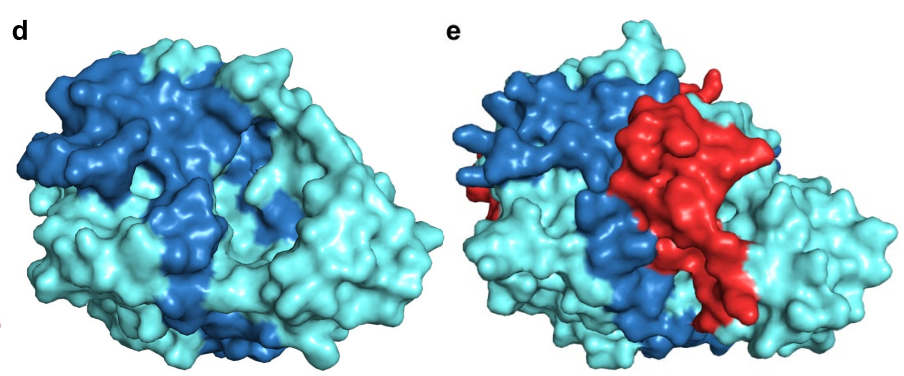

aestivum carboxypeptidase 2; yPRC1, yeast carboxypeptidase Y.b, d, Crystal structure of TaCBP2 (PDB: 1WHT) in cartoon (b) and surface (d) representation showing disulfide bonds (yellow) and internal propeptide removal sites. c, e, Homology model of $A b$ LS based on the crystal structure of TaCBP 2 in cartoon (c) and surface $(\mathbf{e})$ representation showing $\mathrm{N}$-terminal signal peptide (red, bottom right in c), disulfide bonds (yellow), and putative internal propeptide (red, top/middle), which appears to block active site access. Note that surface views in $\mathbf{d}$ and $\mathbf{e}$ are rotated $90^{\circ}$ towards the viewer. 


\section{Article}

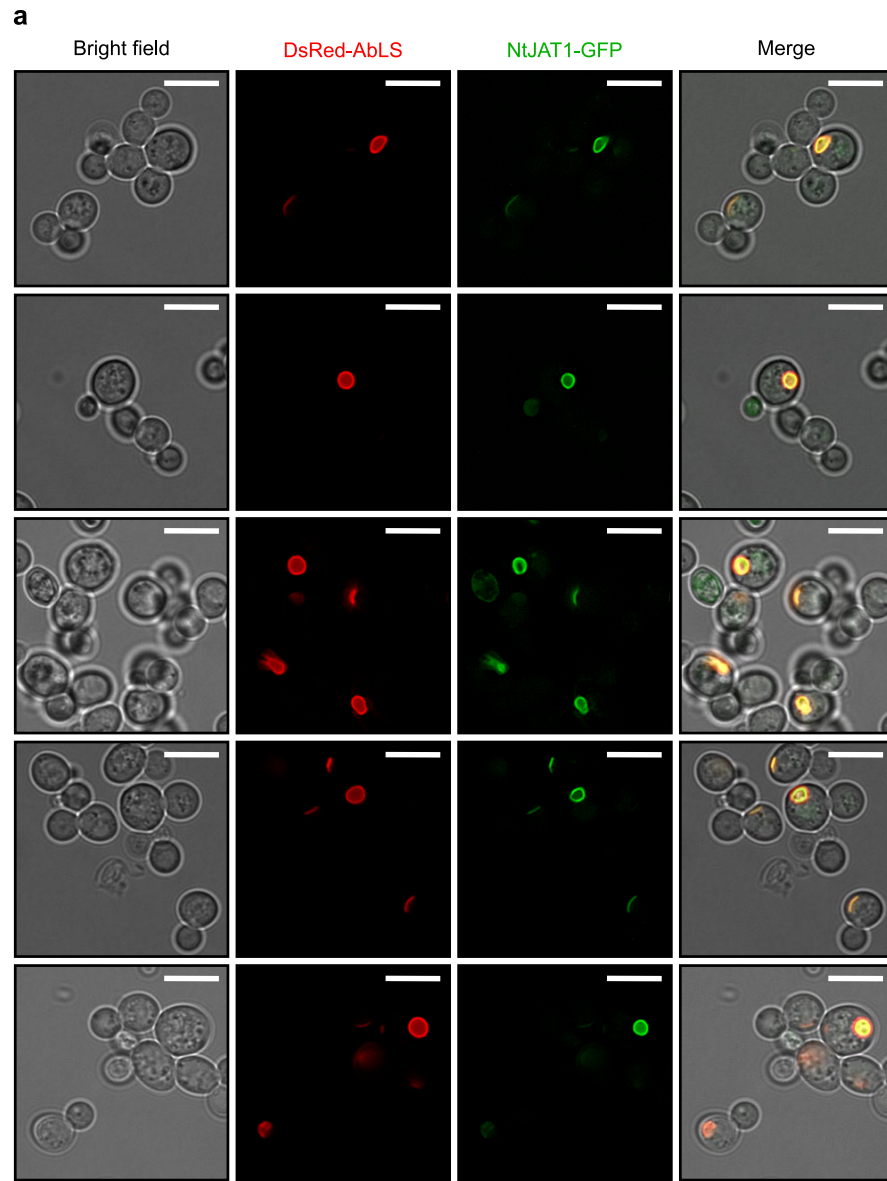

Extended Data Fig. 8 | Fluorescence microscopy of tobacco alkaloid transporters expressed in CSY1296 for alleviation of vacuolar TA transport

limitations. a, b, C-terminal GFP fusions of $N t$ JAT1 (a) and NtMATE2 (b) were expressed from low-copy plasmids in CSY1296. Strains were cultured, imaged,

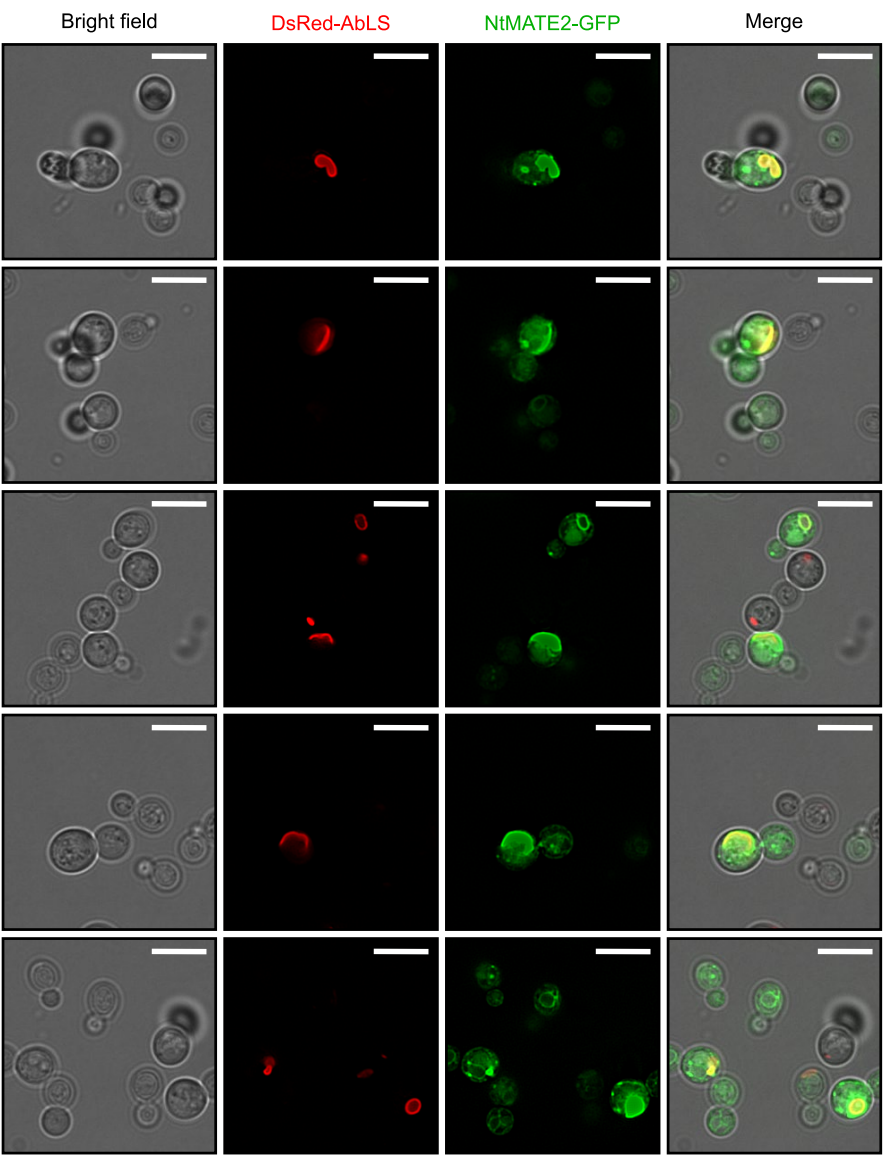

and analysed via widefield epifluorescence microscopy and 2D deconvolution analysis as described in the Methods. Images are representative of two independent experiments. Scale bar, $5 \mu \mathrm{m}$. 

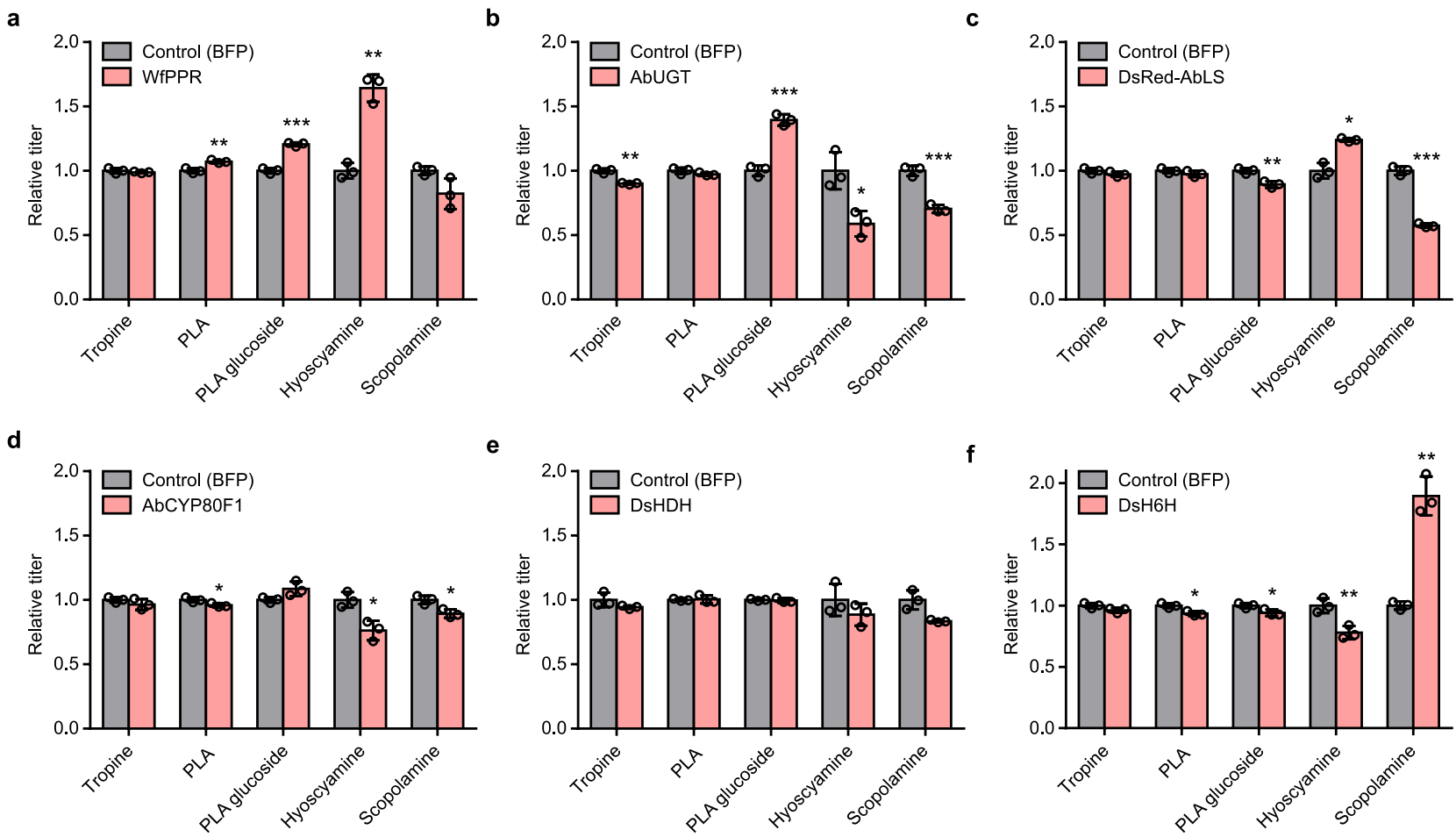

Extended Data Fig. 9 | Effect of extra gene copies on accumulation of TA pathway intermediates and products in scopolamine-producing strain CSY1296. a-f, An additional copy of each biosynthetic enzyme between tropine and scopolamine was expressed from the following low-copy plasmids in strain CSY1296: WfPPR, pCS4436 (a); $A b U G T$, pCS4440 (b); DsRed-AbLS, pCS4526 (c); $A b C Y P 80 F 1, p C S 4438$ (d); DsHDH, pCS4478 (e); DsH6H, pCS4439 (f); or a BFP control (pCS4208, pCS4212, or pCS4213). Transformed strains were cultured in appropriate selective media at $25^{\circ} \mathrm{C}$ for $96 \mathrm{~h}$ before quantification of metabolites in the growth medium by LC-MS/MS analysis of culture supernatant. Note that littorine accumulation was not observed for any samples. Data indicate the mean of $n=3$ biologically independent samples (open circles) and error bars denote s.d. Metabolite titres are shown relative to the BFP control with the same auxotrophic marker as the biosynthetic gene: $W f \mathrm{PPR}(\mathbf{a}), \mathrm{DsRed}-A b \mathrm{LS}(\mathbf{c}), A b \mathrm{CYP} 80 \mathrm{~F} 1$ (d), and $D s \mathrm{H} 6 \mathrm{H}$ (f) relative to the pCS4213 control (LEU2); $A b U G T$ (b) relative to the pCS4208 control (URA3); and $D s \mathrm{HDH}(\mathbf{e})$ relative to the pCS4212 control (TRP1). ${ }^{*} P<0.05,{ }^{* *} P<0.01$, ${ }^{* * *} P<0.001$, Student's two-tailed $t$-test. Statistical significance is shown relative to corresponding controls. (a) PLA, $P=0.00950$; PLA glucoside, $P=3.36 \times 10^{-4}$; hyoscyamine, $P=0.00221 ;(\mathbf{b})$ tropine,$P=0.00500$; PLA glucoside, $P=3.59 \times 10^{-4}$; hyoscyamine, $P=0.0192$; scopolamine, $P=6.90 \times 10^{-4}$; (c) PLA glucoside, $P=0.00544$; hyoscyamine, $P=0.0165$; scopolamine, $P=3.43 \times 10^{-4}$; (d) PLA, $P=0.0487$; hyoscyamine, $P=0.0154$; scopolamine, $P=0.0159 ;$ (f) PLA, $P=0.0153$; PLAglucoside, $P=0.0453$; hyoscyamine, $P=0.00958$; scopolamine,$P=0.00816$. 

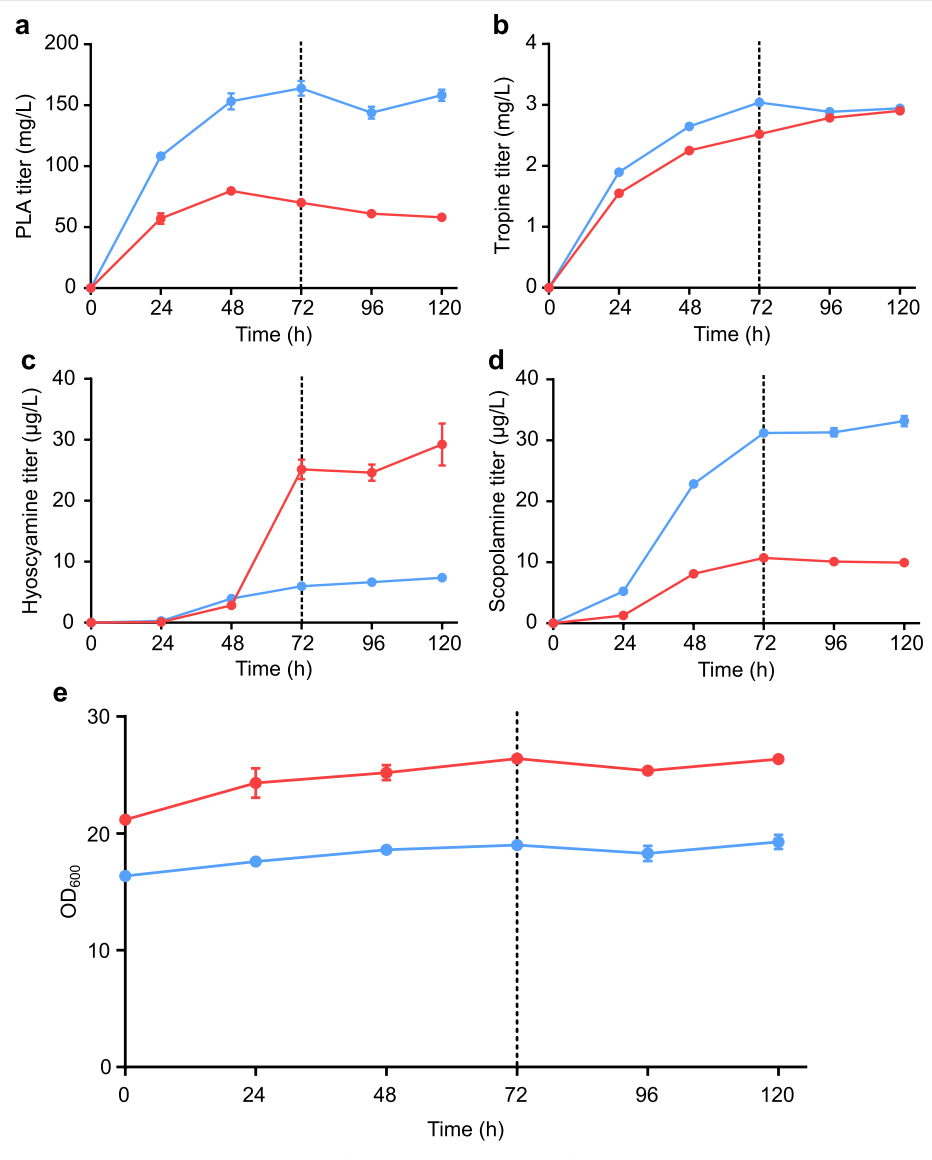

$$
\rightarrow \text { CSY1297 } \rightarrow \text { CSY1298 }
$$

f + ESIMRM $290 \rightarrow 124$

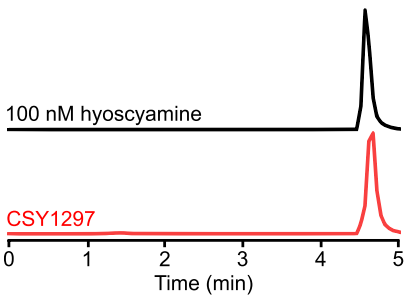

h

+ ESI MRM $304 \rightarrow 138$

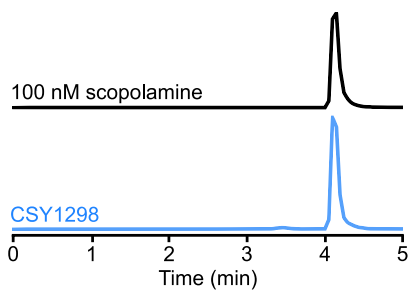

Extended Data Fig. 10 | Time courses of de novo TA and precursor production in pseudo-fed-batch cultures of CSY1297 and CSY1298. a-e, TA-producing strains CSY1297 and CSY1298 (with pCS4213) were respectively cultured in non-selective or selective (leucine dropout) media with $50 \mathrm{mM}$ 2-OG and $15 \mathrm{mgl}^{-1} \mathrm{Fe}^{2+}$ under pseudo-fed-batch conditions at $25^{\circ} \mathrm{C}$ for $120 \mathrm{~h}$. Culture supernatants were sampled for PLA $(\mathbf{a})$, tropine $(\mathbf{b})$, hyoscyamine $(\mathbf{c})$ and scopolamine (d) titres by LC-MS/MS analysis and optical density at $600 \mathrm{~nm}$ $\left(\mathrm{OD}_{600}\right)(\mathbf{e})$ every $24 \mathrm{~h}$. Cultures were supplemented with additional dextrose, glycerol and amino acids to final concentrations of $2 \%, 2 \%$ and $1 \times$ at $72 \mathrm{~h}$ (vertical dotted line). Data indicate the mean of $n=3$ biologically independent g $+E S I M R M 290 \rightarrow{ }^{* *}, C I D @ 20 \mathrm{eV}$

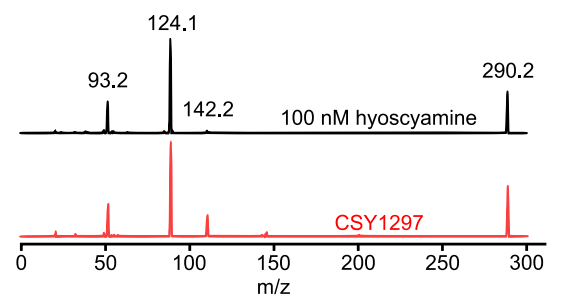

i $\quad+E S I M R M 304 \rightarrow{ }^{* *}, C I D @ 17 \mathrm{eV}$

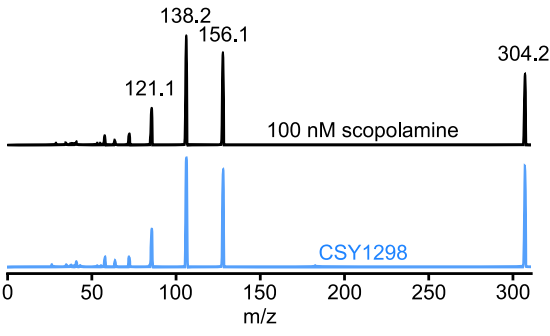

samples and error bars denote s.d. Note that no littorine accumulation was observed in any samples. $\mathbf{f}-\mathbf{i}$, LC-MS/MS verification of de novo medicinal TA production in CSY1297 and CSY1298. Panels show LC-MS/MS chromatograms of hyoscyamine (f, $\mathbf{g})$ and scopolamine $(\mathbf{h}, \mathbf{i})$ detected in CSY1297 and CSY1298 cultures grown in non-selective medium or selective medium, respectively (samples from experiment in c and d, 120 h), and of authentic $100 \mathrm{nM}$ chemical standards. Primary MRM transitions used for detection and quantification are shown in $\mathbf{f}$ and $\mathbf{h}$; additional characteristic transitions are shown in $\mathbf{g}$ and $\mathbf{i}$ ( ${ }^{(* * \prime}$ indicates any detected fragment mass in product ion scan). Traces are representative of $n=3$ biologically independent samples. 


\section{natureresearch}

Corresponding author(s): Christina D. Smolke

Last updated by author(s): Jul 16, 2020

\section{Reporting Summary}

Nature Research wishes to improve the reproducibility of the work that we publish. This form provides structure for consistency and transparency in reporting. For further information on Nature Research policies, see Authors \& Referees and the Editorial Policy Checklist.

\section{Statistics}

For all statistical analyses, confirm that the following items are present in the figure legend, table legend, main text, or Methods section.

n/a Confirmed

$\square \bigotimes$ The exact sample size $(n)$ for each experimental group/condition, given as a discrete number and unit of measurement

$\square$ \A statement on whether measurements were taken from distinct samples or whether the same sample was measured repeatedly

The statistical test(s) used AND whether they are one- or two-sided

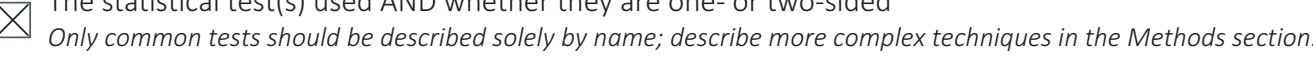

\ $\square$ A description of all covariates tested

$\triangle \square$ A description of any assumptions or corrections, such as tests of normality and adjustment for multiple comparisons

$\triangle$ A full description of the statistical parameters including central tendency (e.g. means) or other basic estimates (e.g. regression coefficient)

$\triangle$ AND variation (e.g. standard deviation) or associated estimates of uncertainty (e.g. confidence intervals)

$\varnothing$ For null hypothesis testing, the test statistic (e.g. $F, t, r$ ) with confidence intervals, effect sizes, degrees of freedom and $P$ value noted Give $P$ values as exact values whenever suitable.

Х $\square$ For Bayesian analysis, information on the choice of priors and Markov chain Monte Carlo settings

Х $\square$ For hierarchical and complex designs, identification of the appropriate level for tests and full reporting of outcomes

Х $\square$ Estimates of effect sizes (e.g. Cohen's $d$, Pearson's $r$ ), indicating how they were calculated

Our web collection on statistics for biologists contains articles on many of the points above.

\section{Software and code}

Policy information about availability of computer code

Data collection Agilent MassHunter Workstation LC/MS Data Acquisition for 6400 Series Triple Quadrupole software (ver. B.08.02) was used for collection of LC-MS/MS data. Agilent MassHunter Workstation Optimizer for 6400 Series Triple Quadrupole software (ver. B.08.02) was used for development of multiple reaction monitoring parameters for chemical standards. Zen Pro software (Blue edition) was used for acquisition of microscopy data. NCBI BLAST online software (ver. 2.8.1) was used for alignment of DNA and amino acid sequences. RaptorX online software (ver. 2018) was used for construction of template-based homology models of protein structures.

Data analysis

$$
\begin{aligned}
& \text { Agilent MassHunter Workstation Qualitative Analysis Navigator software (ver. B.08.00) was used for analysis of all LC-MS/MS data. } \\
& \text { Microsoft Excel } 2013 \text { and Graphpad Prism } 7 \text { were used for analysis and statistical evaluation of all quantitative data, and for preparation } \\
& \text { of figures. NIH ImageJ software (ver. 1.52) was used for analysis of microscopy data, along with the "Diffraction PSF 3D" (ver. } 6 \text { June } \\
& \text { 2005) and "Parallel Spectral Deconvolution" (ver. 1.9) plugins. PyMOL software (ver. 2.2.3) was used for visualization and analysis of } \\
& \text { enzyme structures. De novo transcriptome assembly was performed using the publicly available Trinity software package (ver. 2.7.0) and } \\
& \text { the following plugins: SRA Toolkit (ver. 2.9.1), FastQC (ver. 0.11.7), BBTools/BBDuk.sh (ver. 38.16), Trinotate (ver. 3.1.1), HMMER (ver. } \\
& \text { 3.2.1). Inkscape (ver. 0.91) was used for preparation of figure panels. Small molecule geometry optimization was performed using the } \\
& \text { Gaussian software package (ver. 16). Molecular docking simulations were conducted using the Schrodinger Maestro software suite (ver. } \\
& \text { 12.0) and the Glide XP plugin (ver. 8.3). Phylogenetic sequence analyses were performed using ClustalX2 (ver. 2.1) and visualized using } \\
& \text { FigTree (ver. 1.4.3). The custom R script used for coexpression analysis of plant RNA sequencing data has been deposited in, and is } \\
& \text { available from, a public GitHub repository at github.com/smolkelab/Oxidoreductase_coexpression_analysis. }
\end{aligned}
$$


Policy information about availability of data

All manuscripts must include a data availability statement. This statement should provide the following information, where applicable:

- Accession codes, unique identifiers, or web links for publicly available datasets

- A list of figures that have associated raw data

- A description of any restrictions on data availability

Data supporting the findings of this work are available within the paper and its Supplementary Information files. The datasets generated and analyzed during the current study are available from the corresponding author upon reasonable request. This article includes raw source data files associated with Figs. 1-4 and Extended Data Figs. 3, 4, 6 (Supplementary Figure 1), 9, and 10. Novel genetic sequences identified and characterized in this study are available via the following accession codes from public databases. 1000Plants (1KP) database: scaffold-AIOU-2012986-Brugmansia_sanguinea (BsUGT); scaffold-JNVS-2051323-Datura_metel (DmUGT). Medicinal Plant RNAseq database: medp datin_20101112/6354 (DiHDH); medp datst_20101112|10433 (DsHDH). MSU Medicinal Plant Genomics Resource: full amino acid sequences and database accession numbers (IDs) for all tested HDH candidates are provided in Supplementary Table 1. Accession numbers for previously reported gene and protein sequences in the GenBank/UniProt databases are provided in Supplementary Table 2. Protein crystal structures used for homology modeling are available from the RCSB protein data bank: Arabidopsis thaliana salicylate UDP-glucosyltransferase UGT74F2 with bound UDP, PDB: 5V2K; Populus tremuloides sinapyl alcohol dehydrogenase with bound NADPH, PDB: 1YQD. The custom R script used for identification of HDH candidates via coexpression analysis of RNA sequencing data is available from the Smolke Laboratory GitHub: github.com/smolkelab/Oxidoreductase_coexpression_analysis.

\section{Field-specific reporting}

Please select the one below that is the best fit for your research. If you are not sure, read the appropriate sections before making your selection. $\bigotimes$ Life sciences $\quad \square$ Behavioural \& social sciences $\quad \square$ Ecological, evolutionary \& environmental sciences

For a reference copy of the document with all sections, see nature.com/documents/nr-reporting-summary-flat.pdf

\section{Life sciences study design}

All studies must disclose on these points even when the disclosure is negative.

Sample size All presented titer data represent measurements from sample sizes of $n=3$ biological replicates, where biological replicates (as defined in 'Replication' below) represent independently grown microbial cultures. Since (i) engineering was performed at the cellular level, (ii) metabolite titers are a bulk measure of a cellular population, and (iii) each assayed microbial culture represents a large population of individual cells, $n=3$ biological replicates were chosen as they are sufficient for reliable measurement of changes in metabolite production at the population level and for statistical analyses, based on previous metabolic engineering papers.

Data exclusions No data were excluded from the analyses.

Replication All measurements of metabolite titers were performed on $n=3$ biologically independent samples (biological triplicates). All replicates performed in this study were biological replicates, rather than technical replicates, and represent independent data points. For example, replicate microbial cultures were grown in separate containers/wells and assayed independently from one another. Fluorescence microscopy and Western blot analyses shown in the paper are representative of two or three independent experiments; all attempts at replication of results presented in this study were successful.

Randomization Randomization of individual cells was not relevant to this study, as the biological subjects for assays were bulk cultures of microbial cells. Microbial strains used for all experiments were inoculated from randomly selected colonies on agar plates. Representative fields of view were selected randomly for fluorescence microscopy.

Blinding Complete blinding was not feasible for this study, as samples were prepared and measured by the same researcher. However, all samples were labeled with numeric codes, rather than names or descriptions, throughout the sample preparation and data collection work-flow to simulate blinding.

\section{Reporting for specific materials, systems and methods}

We require information from authors about some types of materials, experimental systems and methods used in many studies. Here, indicate whether each material, system or method listed is relevant to your study. If you are not sure if a list item applies to your research, read the appropriate section before selecting a response. 


\begin{tabular}{l|l}
\hline n/a & Involved in the study \\
$\square$ & $\bigotimes$ Antibodies \\
$\square$ & $\square$ Eukaryotic cell lines \\
$\square$ & $\square$ Animals and other organisms \\
$\square$ & $\square$ Human research participants \\
$\square$ & $\square$ Clinical data
\end{tabular}

\begin{tabular}{l|l}
\hline n/a & Involved in the study \\
$X$ & $\square$ ChIP-seq \\
$X$ & $\square$ Flow cytometry \\
$X$ & $\square$ MRI-based neuroimaging
\end{tabular}

\section{Antibodies}

Antibodies used

Validation
Rabbit IgGk anti-HA HRP-conjugated antibody (Absolute Antibody, 16.43/Ab00828-23.0)

As stated on the datasheet for this product on the manufacturer's website, the rabbit IgGK anti-HA HRP-conjugated antibody was validated for use in Western blot (as well as other applications). A rat IgG2a version of this antibody clone (16.43), which shares the same variable domain sequences as the rabbit IgGk chimeric antibody used here, has been used in a previous study for immunohistochemical staining of rat YB2/0 hybridoma cells (see manufacturer's website for reference).

\section{Eukaryotic cell lines}

Policy information about cell lines

Cell line source(s)

Authentication

Mycoplasma contamination

Commonly misidentified lines (See ICLAC register)
Wild-type Saccharomyces cerevisiae strain CEN.PK2-1D was obtained from EuroSCARF (30000B).

The commercial cell line used in this study (i.e., Saccharomyces cerevisiae CEN.PK2-1D) was not independently authenticated by the authors of this study. Engineered yeast strains were authenticated by DNA sequencing of relevant genomic modifications, as described in the Methods section of the study.

Not applicable.

Not applicable. 